\title{
International migration and caesarean birth: a systematic review and meta-analysis
}

\author{
Lisa Merry ${ }^{1 *}$, Rhonda Small ${ }^{2}$, Béatrice Blondel ${ }^{3}$ and Anita J Gagnon ${ }^{4}$
}

\begin{abstract}
Background: Perinatal health disparities including disparities in caesarean births have been observed between migrant and non-migrant women and some literature suggests that non-medical factors may be implicated. A systematic review was conducted to determine if migrants in Western industrialized countries consistently have different rates of caesarean than receiving-country-born women and to identify the reasons that explain these differences.

Methods: Reports were identified by searching 12 literature databases (from inception to January 2012; no language limits) and the web, by bibliographic citation hand-searches and through key informants. Studies that compared caesarean rates between international migrants and non-migrants living in industrialized countries and that did not have a 'fatal flaw' according to the US Preventative Services Task Force criteria were included. Studies were summarized, analyzed descriptively and where possible, meta-analyzed.
\end{abstract}

Results: Seventy-six studies met inclusion criteria. Caesarean rates between migrants and non-migrants differed in $69 \%$ of studies. Meta-analyses revealed consistently higher overall caesarean rates for Sub-Saharan African, Somali and South Asian women; higher emergency rates for North African/West Asian and Latin American women; and lower overall rates for Eastern European and Vietnamese women. Evidence to explain the consistently different rates was limited. Frequently postulated risk factors for caesarean included: language/communication barriers, low SES, poor maternal health, GDM/high BMl, feto-pelvic disproportion, and inadequate prenatal care. Suggested protective factors included: a healthy immigrant effect, preference for a vaginal birth, a healthier lifestyle, younger mothers and the use of fewer interventions during childbirth.

Conclusion: Certain groups of international migrants consistently have different caesarean rates than receivingcountry-born women. There is insufficient evidence to explain the observed differences.

Keywords: Caesarean, Immigrants, Refugees, Risk factors, Meta-analysis

\section{Background}

The use of medical interventions in birth has quickly risen in the last quarter century particularly in industrialized countries where the development of medical technology has advanced rapidly [1]. Most notable has been the dramatic rise in caesarean births with rates in the last 15 years in the US, Canada, Australia and parts of Europe reaching 25\% and above (see http://www.oecd.org/els/healthsystems/oecdhealthdata2012-frequentlyrequesteddata.htm). While a caesarean birth can be a life-saving procedure it is associated with a significantly increased risk of maternal

\footnotetext{
* Correspondence: lisa.merry@mail.mcgill.ca

'Ingram School of Nursing, McGill University, Montreal, QC, Canada

Full list of author information is available at the end of the article
}

death from complications of anaesthesia, puerperal infection, and venous thromboembolism [2]. The World Health Organization therefore recommends that caesareans be performed only in medically necessary instances [3].

Migration flows are increasing globally [4] and migrants contribute substantially to the total number of births, exceeding in some Western countries one fifth of births $[5,6]$. The health of migrants, including perinatal health, has therefore become a research priority [7]. Migrants (immigrants, refugees, asylum-seekers, undocumented and others with temporary or irregular statuses) [8,9] face multiple and intersecting social determinants of health that may compound childbearing health risks, including lower social economic status, lack of support, high levels of stress

\section{Biomed Central}


related to migration and resettlement and barriers in accessing healthcare $[10,11]$. Refugees and asylum-seekers may also have suffered trauma and abuse [12,13]. Perinatal health disparities [14], including disparities in caesarean births have been observed between migrants and receivingcountry-born women and some literature suggests that non-medical factors such as communication barriers, support and/or care practices during labour and delivery, female genital cutting, or cultural preference may also be implicated in caesarean rate differences [15-17]. We conducted a systematic review to address the questions: 1) Do international migrant women in Western industrialized countries have consistently different rates of caesarean birth than receiving-country-born women? And 2) What are the mechanisms (medical and/or non-medical factors) that might explain differences?

\section{Methods}

The "Meta-analysis of Observational Studies in Epidemiology (MOOSE)" recommended guidelines for the publication of meta-analyses of observational studies were used to prepare this manuscript [18].

We searched for cross-sectional and cohort studies that provided comparisons of caesarean rates between migrant and non-migrant women. To be included in the review, studies must have examined migrants who crossed international borders and who were living in an industrialized country, defined using the Organization for Economic Cooperation and Development (OECD) member list; studies conducted in refugee camps or with internally displaced persons were excluded. No restrictions were applied based on migrant origin, status or length of time in receivingcountry. We searched the following electronic citation databases: Medline, Health Star, Embase, PsycInfo, CINAHL, Sociological Abstracts, Web of Science, Proquest Research Library, Proquest Dissertations and Theses, POPLINE, Global Health and PAIS. The search strategy was developed in conjunction with a McGill University Health Sciences librarian and can be found in Additional file 1. No language limitations were applied. Searches were conducted from inception of each database until January 2012.

We also hand-searched reference lists of identified literature, conducted web searches and contacted migration and health research experts for additional literature. Web sites searched included professional agency [(e.g., International Federation of Gynecology and Obstetrics (FIGO)] and government (Canada, US and Australia) health sites (see Additional file 2). Each site was searched by browsing through relevant indices predefined by each website and by using 'search boxes'. Terms included: "c(a)esarean, csection, mode of delivery, (im)migrant, (im)migration, foreign, country of birth". Requests for literature were made through ROAM (Reproductive Outcomes and Migration), an international research collaboration, involving over 30 researchers from 13 countries, including Canada, Australia and Europe (see http://migrationandreproductivehealth.org/?page_id=76).

We reviewed all abstracts (or full articles if necessary) to determine if inclusion criteria were met. Those included were subsequently fully reviewed and quality-assessed and data were extracted into a database in $\operatorname{Excel}^{\odot}$. NonEnglish language reports were reviewed by ROAM collaborators and if no researcher could read the language of the report, we reviewed the English version abstract only.

The US Preventative Services Task Force criteria were used to assess the quality of studies [19]. Studies were evaluated on the 1) comparability of the study groups (assembly and maintenance); 2) degree of participant loss; 3) clarity of their "migrant" and "non-migrant" classifications; 4) validity and reliability of the approaches used to measure the migrant, caesarean birth and other variables; and 5) attention given to potential confounding factors (i.e., age and parity). Studies with a "fatal flaw" (i.e., $\geq 50 \%$ of the original sample lost to analysis and/or comparison groups not adequately defined) were removed from further analysis. Studies were scored as either "good" or "fair"- the former if the study met all quality criteria and the latter if it did not meet all criteria but had no fatal flaw. One reviewer assessed all studies and a second reviewer independently assessed 25\% of the studies to confirm the assigned quality scores. Discrepancies were resolved through discussion.

A standardized coding form was developed to extract three types of data: 1) publication year and language; 2) study characteristics (e.g., study sample and data sources) and 3) results. The migration indicator (i.e., how migrants were labelled in the study) was recorded verbatim from the report and categorized by type as: "source country/region", "foreign-born/non-national", "ethnicity", "length of time" or "migration status". Sources of data were classified as: 1) population-based registry; 2) population-based hospital data; 3) population-based survey; or 4) hospital records/research study. Timeframe and geographical coverage (local, regional, or national) of data collection, host country and number of migrants and non-migrants were also recorded. Extracted results included frequencies of caesarean rates (overall, emergency and elective) and indications for caesarean for migrants and non-migrants separately. Explanations for caesarean rate differences between groups postulated in discussion sections were also extracted.

For each study we defined caesarean rates for migrant women as being "higher", "lower" or "not different" compared to non-migrant women. If a study had results reported for more than one migrant group comparison they were coded as: "higher" if migrant results in all comparison groups were higher or a combination of higher and not different; "lower" if all were lower or 
lower and not different; "mixed" if some were higher and some lower; and "not different" if all rates between the migrant and non-migrant groups were not statistically different.

We used Review Manager $5.1^{\odot}$ to perform metaanalyses to estimate the 'effect' of being a migrant on the outcomes of interest: overall caesarean and emergency and elective caesarean rates. The studies were assumed to be heterogeneous due to the range of host countries and migrant groups examined and changes in obstetrical practice over time. We therefore used a random effects model which regards an effect as variable across studies and produces more conservative effect estimates with wider confidence intervals [20]. We combined and analyzed migrant sub-groups defined using UN macro-regions (see Additional file 3) or country of origin, migration status, and length of time in receivingcountry. Sub-analyses by parity, receiving- country/region and time period of data collection were also performed. Due to the diversity across studies in variables selected to control for confounding we chose to use unadjusted results in meta-analyses. We calculated each study's effect size as an odds ratio (OR) using the Mantel-Haenszel model (event/total for migrants and receiving-country women respectively) [20]. Attention was given to ensure duplicate data from different reports were not included twice in analyses and only one comparison from each study was combined for metaanalyses to ensure the results from a comparison group were not counted more than once. A weighted summary effect was calculated for each analysis performed.

To investigate the robustness of results which showed migrant women to consistently have different rates of caesarean compared to receiving-country-born women, sensitivity analyses were performed by limiting the analyses to only population-based data. To assess whether results would differ when covariates were adjusted, we also performed analyses by using adjusted odds ratios and applying the generic inverse variance method to produce summary estimates. Heterogeneity for all metaanalyses was assessed using $\mathrm{I}^{2}$ tests and we considered a value of $50 \%$ or more to indicate substantial heterogeneity [20]. We also examined confidence intervals among the studies in each forest plot to assess the degree of overlap; less overlap being interpreted as evidence of heterogeneity [20]. Publication bias was assessed by visual examination of funnel plots of the OR against the standard error of the log of the OR of studies within each migrant subcategory [20].

\section{Results}

The database search yielded over eight thousand citations $(n=8565)$. Once duplicates and studies that did not meet the inclusion criteria were excluded, 87 reports remained. Seventeen additional reports were identified: seven through hand searches, nine from ROAM collaborators and one annual report published by the New South Wales (Australia) Department of Health [21] online. Three reports could not be reviewed because of language [22-24]. Twenty studies were removed because the comparison group included migrants, and/or the migrant group included native-born, and/or the assembly of the migrant and non-migrant groups were dissimilar. Seventy-six studies met the inclusion criteria and were assigned a quality score (see Table 1 for a complete description of included studies) [15-17,25-103]. Twentytwo studies were scored as good and 54 were scored as fair (see Table 2). The primary reasons for not meeting all of the quality criteria was a lack of attention to confounding and ambiguity on how study groups (migrants vs. non-migrants) were defined or confirmed.

Across the 76 studies, data were reported on 1,029,454 migrants (see Table 2). The most common source regions represented were Latin America \& Caribbean (39\%) followed by 'origin unspecified' (11\%) and South Asia (7\%) (see Table 2). Region and country of origin were the migration indicators most often used to report caesarean rates (57 of 76 studies). "Foreign-born" was used in 16 studies; ethnicity/race/religion/language, length of time and migration status (e.g., "refugees"), were also used in five, three and nine studies respectively.

The receiving countries included Europe (68\%), Australia (11\%), the US (11\%), Canada (6\%) and Israel (4\%). The primary language of publication was English (82\%). Data sources were population-based in $36 \%$ of studies, and coverage for the majority (65\%) of studies was one city (in most cases one hospital). Data were gathered from 1956 to 2010; 70\% from the 1990s or later. More than half of the studies were published from 2000 onward (see Table 2).

\section{Caesarean Birth Rates: Migrants vs. Non-migrants}

Comparison of caesarean rates overall between migrants and non-migrants revealed that in $69 \%$ of the studies there were differences in caesarean rates between the two groups (see Table 2). For emergency caesareans, more than half of the studies showed migrants to have higher rates or higher and no different compared to receiving-country-born whereas for elective caesareans there was more variation: $26 \%$ showed higher rates or higher and no different; $37 \%$ showed lower rates or lower and no different and 5\% of studies had mixed results (see Table 2).

\section{Meta-analyses}

\section{Sub-Saharan Africa (or Africa unspecified)}

African migrants had an excess of caesareans compared to receiving-country-born women: France $[\mathrm{OR}=2.22$, 
Table 1 Description of included studies

\section{Reference (language of publication)}

Alonso (P, Maresca MI, Oritz TA, \& Serrano

MM. (2006) (Spanish)

Aurelius G, \& Ryde-Blomqvist E. (1978) (English)

Barron SL, \& Vessey MP. (1966) (English)

Berger C, Liska G, Gallier J, \& Soutoul JH (1973) (French)

Berger C, Laugier J, \& Soutoul JH. (1974) (French)

Bona G, Zaffaroni M, Cataldo F, Sandri F, \& Salvioli GP. (2001) (English)

Braveman $\mathrm{P}$, Egerter $\mathrm{S}$, Edmonston $\mathrm{F}$, \& Verdon M. (1995) (English)

Cassell E. (1995) (English) Thesis

Chan A, Roder D, \& Macharper T. (1988) (English)

Comas M, Català L, Sala M, et al. (2011) (English)
Method

(geographica

coverage, data

source, data yr)

Local, hospital
records, $2000-$

2002

Local, hospital records, 1968-

1969

Local, hospita records, 1958-

1960

Local, hospital records, 1970-

1972

National,

population-

based hospital data, 1996-1997

Regional,

population-

based registry

1991

Regional,

population-

based registry

1982-1992

Regional,

population-

based registry

1981-1983

Local, hospital

records, 2006

2007

\section{Receiving country}

Population

Sweden (Stockholm)

UK (South London)

Italy

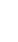

US (California)

Australia (Victoria)

Australia (South Australia)

Spain (Barcelona)

included.

women. women.
Findings

vs. Non-migrants*

(Everall caesarean rates) (Emency

\&/or Elective caesarean rates)

2759 African, American, European, \& Oriental L \& ND overall (not statistically tested)

Fair

"immigrants" vs. 3990 Spanish women.

1235 "Immigrants" from Scandinavia, West,

South, \& East Europe, \& "other countries" vs.

412 Swedish women. Only singleton births

H \& ND overall (unadjusted)

1563 West Indian \& Irish "immigrants" vs. 3891 H overall (not statistically tested; W Indian: British women. Only singleton births. primips $\mathrm{H}$, multips ND; Irish: primips ND, multips $\mathrm{H}$ )

800 Portuguese/Spanish, North African,

Yugoslavia, "Other" "migrants" vs. 2655 French

M overall (not statistically tested)

3347 "Legal immigrants from developing

countries" (Latin America, North Africa, Sub

Saharan Africa, Middle East, Indian Sub-

ND overall (all immigrants combined: unadjusted; Variation by groups but not statistically tested)

Gipsies) vs. 6694 Italians.

81,445 Asian and Latina "foreign born" vs.

93,685 "Whites". Only singleton primiparous

L \& ND overall (adjusted)

5268 Filipino-born vs. 507,457 Australian-born. H overall (stratified by parity and age caesarean rates consistently $\mathrm{H}$ ) $\mathrm{H}$ emerg (unadjusted) $\mathrm{H}$ elect (unadjusted)

5675 "Non-English speaking countries" (includes Italy, Holland, Germany, Vietnam, Greece, Yugoslavia, Philippines, \& "other") vs. 2894 Australian-born.

H overall (all immigrants combined unadjusted; Variation by groups but not statistically tested) $\mathrm{H}$ emerg (all immigrants combined; unadjusted) $\mathrm{H}$ elect (all immigrants combined; unadjusted)

564 Foreign-born (includes women from South America, Asia and North AfricaND overall (all immigrants combined; unadjusted)

Morocco, Pakistan and Ecuador most

represented) vs. 462 Spanish-born women.

Only women who lived in hospital catchment area were included. 
Belgium (Liege)

89 North-African nationality vs. 184 Belgian

ND overall (unadjusted)

nationality women. Singleton live births,

excluded women with gestational diabetes \&

Diani F, Zanconato G, Foschi F, Turinetto A, \& Local, hospital Franchi M. (2003) (English) records, 1992200

Fedeli U, Alba N, Lisiero M, Zambon F, Avossa Regional, F, \& Spolaore P. (2010) (English) populationbased registry 2006-2007

Forna F, Jamieson DJ, Sanders D, \& Lindsay MK. (2003) (English)

Local, hospital records, 1991200

Gagnon AJ, Dougherty G, Platt RW, et al. (2007) (English) and Gagnon AJ, Van Hulst Merry L, et al. (2012) (English) Unpublished when review of literature was conducted, 2007 reference describes original study; 2012 reference is publication of caesarean results (after review of literature was completed)

Gagnon AJ, Wahoush O, Dougherty G, et al. (2006) (English) Unpublished, 2006 reference describes study

Gann P, Nghiem L, \& Warner S. (1989) (English)

Gayral-Taminh M, Arnaud C, Parant O, Fournie A, Reme JM, \& Grandjean H. (1999) (French)

Local, research Canada (Montreal, Toronto study, 2003-2004 Vancouver) ( Low-risk population) ND emerg (unadjusted

Local, research Canada (Montreal, Toronto, 1025 "Recently arrived migrants" ( $\leq 5$ years)

Local, hospital US (Lowell) records, $1981-$ 1987

Local, hospital France (Toulouse) records, 19881994 (excl

Giani U, Bruzzese D, Pugliese A, Saporito M, \& Regional, Triassi M. (2011) (Italian, used English abstract) population2005

Harlap S, Kaufman R, Prywes R, Davies AM Sterk W, \& Weiskopf P. (1971) (English)
Local, research study, 1964-1967
Italy (Verona)

1014 "Non-EU women" (including Central Africa, Northern Africa/Middle East, Eastern Europe, Asia, \& Latin America) vs. 12,931 Italians.

Italy (Veneto region)

20,332 Regular and Irregular foreign born/ migrants vs. 73,098 Italian women.

\section{US (Atlanta)}

13,465 Africa, Asia, Caribbean, Europe, Centrat \& South America, Middle East \& "other"

foreign-born vs. 36,439 US born (mostly Black women). Excluded women with no prenatal care.

1018 "Recently arrived migrants ( $\leq 5$ years)" and defined by UN Macro Region (Europe,

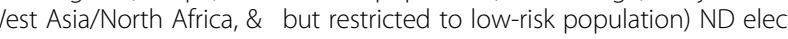
South-Central Asia), migration status (refugee, (not statistically tested) asylum seeker, non-refugee immigrant) and length of time ( $\leq 2$ years vs. $>2$ years) vs.

2482 Canadian-born. Only low-risk $<35$

years, gestational age $\leq 42$ weeks)

primiparous women defined by migration status: refugee, asylum seeker, non-refugee immigrant vs. 514 Canadian-born.

H overall (all immigrants combined; unadjusted)

L overall (unadjusted; when stratified by age for regular migrants ND across strata except ages 30-34 years $\mathrm{H}$ )

H \& ND overall (unadjusted)

310 Cambodian refugees vs. 110 low income Whites. Only singleton births included.

Primary caesareans only.

2636 "Black Africa" \& Maghreb vs. 3172 French women. Only singleton births included.

\& ND overall (unadjusted; results consistent when stratified by parity) ND during labour caesarean (unadjusted) ND pre-labour caesarean (unadjusted)

Italy (Campania)

1709 Foreign-born women vs. 28,557 Italian L elect (adjusted)

women. Excluded repeat caesareans.

13,112 Asian, North African, \& Western countries (based on birth place) vs. 7635

H overall (not statistically tested) 
Table 1 Description of included studies (Continued)

Hazekamp JT. (1982) (English) Local, hospita

records, 1978

Norway (Oslo)

51 Wives of Pakistani migrant workers vs. 51

Norwegian women matched for age and

parity.

Helsel D, Petitti DB, \& Kunstadter P. (1992) Regional, (English)

Henry OA, Guaran RL, Petterson CD, \&

Walstab JE. (1992) (English)

Holan S, Vangen S, Hanssen K, \& StrayPedersen B. (2008) (Norwegian)

Howell R. (1989) (English)

Ismail KI, Marchocki Z, Brennan DJ, \& O'Donoghue K. (2011) (English)

Ismail Kl, Marchocki Z, Brennan DJ, \&

O'Donoghue K. (2010) (English) Conference abstract

Janevic T.(2011) ${ }^{\dagger}$ (English) Conference abstract Local

Johnson EB, Reed SD, Hitti J, \& Batra M. (2005) (English)

Kaminski M. (1975) (French)
Local, hospital records, 1980198

Local, hospital records, 2009

population-

based registry,

1995-2003

population-

based registry

Local, hospital ecords, 1979-

988

cal, research

study, 1993-1998

US (Merced \& San Joaquin

937 Hmong refugees vs. 3776 non-Hispanic

Whites. Mixed marriages excluded. Only

singleton births included.

Australia (Melbourne)

1123 Vietnamese (likely refugees) vs. 35,373 Australian born women.

220 Asian/African women combined

(including women from Pakistan, Sri Lanka, India, Vietnam, Morocco, \& Somalia)

immigrants with diabetes vs. 262 Norwegian women with diabetes. Only singleton births included.

338 Vietnamese refugees and Filipino women (most married to Caucasian men) vs. 14,790 Australian-born women

Ireland (Cork)

867 Eastern European women (refers to women from Belarus, Bulgaria, Czech Republic, Hungary, Moldova, Poland, Romania, when stratified by age) \& ND (multips, Russia, Slovakia, \& Ukraine); nationality used to unadjusted) caesareans during labour categorize; most would be economic

migrants. Multiple, breech and elective or pre-

labour caesareans were excluded vs. 5550

Irish women.

US (New York)

511,564 "Foreign-born and ethnicity/region of M overall (adjusted)

origin" : North Africa, Sub-Saharan Africa,

Africa unspecified, Non-Hispanic Caribbean,

Hispanic Caribbean, Mexico, South America, Central America, East Asia, South East Asia/ Pacific Islands, \& South Central Asian vs. 449,817 US born women. Singleton birth only.

Regional,

population-

based registry

1993-2001

Local, research France (Paris) study, 1963-1969 Europe \& the Antilles (only women whose
US (Washington)

579 Somali immigrants vs. 4837 US-born Whites \& Blacks. Only singleton births.

1795 Migrants from North Africa, South husbands were also born outside of France were considered migrants) vs. 5774 French women.
ND overall (unadjusted: ND for primips, $L$ \& $\mathrm{ND}$ for multips) $\mathrm{H}$ emerg (not statistically tested), H (primips) \& ND (multips) emerg (unadjusted) L \& ND elect (unadjusted but consistent results by parity)

ND caesareans during labour (unadjusted) L unadjusted) casareans during labour

overall (unclear if statistically significant; results similar when just primips) ND emerg (not statistically tested) ND elect (not

$H$ (compared to US Whites) \& ND (compared to US Blacks) (not statistically tested) H (primips) \& ND (multips) overall; (adjusted) 
Table 1 Description of included studies (Continued)

\begin{tabular}{lll}
\hline Kingston D, Heaman M, Chalmers B, & National, & Canada \\
Kaczorowski J, et al. (2011) & \\
& Population- & \\
& based survey, \\
& 2006 &
\end{tabular}

Lansakara N, Brown SJ, \& Gartland D. (2010) (English)

LeRay C, Carayol M, Zeitlin J, Breart G, \& Goffinet F. (2006) (English)

Loew D, \& Schrank P. (1966) (German)

Ma J, \& Bauman A. (1996) (English)

Malin M, \& Gissler M. (2009) (English)

Maslovitz S, Kupferminc MJ, Lessing JB, \& Many A. (2005) (English)
Local, research study, 2003-2005

National 2001-2002

Local, hospital records, 1956-

1965

Regional,

population-

based registry

1990-1992

National,

population-

based registry

1999-2001

Local, hospital

records, 2001-

2002 research study Wales)

Finland
16,040 "Landed immigrants" (residents)

(including North America, Central America,

Caribbean/Bermuda, South America, West

Europe, East Europe, North Europe, South/

unspecified Europe, East Africa, North Africa,

West/Central Asia/Middle East/Kurdistan, East Asia, South East Asia/Asia/East Timor, South Asia/Asia non-specified) defined as recent ( $\leq$ 5 years) and non-recent arrival (> 5 years) vs. 57,800 Canadian-born. Only singleton births. Weighted numbers.

212 Mothers born overseas of non-English speaking background- needed to be fluent in English to participate; included women from 53 different countries, largest group were

from South Asia, Sri Lanka and India vs. 1074 Australian-born mothers. Primiparous women only.

618 "Origin abroad" (including North Africa, North European, South European, African \& Asian) vs. 2797 French. Only low risk

primiparous, singleton, cephalic presentation, no induction, Birthweight 2500-4500 g babies, included.

Germany (Russelheim)

398 "Foreigners" (including Southern Europe East Europe \& "other") vs. 6602 German women.

Australia (New South

64,922 Immigrant women from Europe, Asia, Africa, New Zealand/Oceania, Middle East America vs. Australian (non-Aboriginal).

6532 Migrants from Latin American/ Caribbean, Somali, African, Vietnamese, South East Asian, Iran/Afghan/Iraq, Chinese, South Asian, Middle East, Baltic, Soviet Union, Eastern Europe, Western Europe, \& Nordic (only those with resident status) vs. 158,469 Finnish women. Only singleton births.

721 Non-resident foreign labourers [mostly Eastern Europe, also included women from Africa (Kenya, Nigeria, Ghana) and Asia

(Thailand, Philippines, China)] vs. 16,012 Israeli residents.
ND overall (all countries combined \& stratified Good by time since arrival; adjusted; Variation by country but not statistically tested)

ND overall (all countries combined; unadjusted) ND emerg (all countries

combined; unadjusted) ND elect (all countries combined; unadjusted)

H caesarean during labour (all migrants combined; adjusted; Variation by groups but not statistically tested)

H overall (all foreigners combined; unadjusted)

M overall (unadjusted)

$M$ overall (unadjusted but results presented by parity)

H overall (all migrants combined; unadjusted) H emerg (all migrants combined; unadjusted) L elect (all migrants combined; unadjusted) 
Table 1 Description of included studies (Continued)

\begin{tabular}{lll}
\hline Merten S, Wyss C, \& Ackermann-Liebrich U. & National, & Switzerland \\
(2007) (English) & population- & \\
& based hospital \\
& data, 2000-2002
\end{tabular}

Moscioni P, Romagnoli C, Pomili G, \& Gilardi G. (1995) (Italian)

Mossialos E, Allin S, Karras K, \& Davaki K. (2005) (English)

Oliva GC, Zannella MP, Filidi C, Cavaliere AF, Casarella L, \& Mancuso S. (2007) (Italian)

Panagopoulos P, Tsoukalos G, Economou A, et al. (2005) (English)

Parsons L, Macfarlane AJ, \& Golding J. (1993) (English)

Press F, Katz M, Leiberman JR, Shoham I, \& Glezerman M. (1993) (English)

Richman D, \& Dixon S. (1985) (English)

Rio I, Castelló A, Barona C, et al. (2010) (English)

Rizzo N, Ciardelli V, Gandolfi-Colleoni G, et al. (2004) (English)

Roman H, Blondel B, Bréart $G$, \& Goffinet $F$. (2008) (English)

Local, hospital records, 1992

1994

Local, hospital records, 2002

Local, hospital records, 2000-

2004

Local, hospital records, 20002004

National,

population-

based registry

1982-1985

Local, hospital records, 1988-

1991

Local, research study, 1980-198

Regional

population-

based registry,

2005-2006

records, 1997

2001

National,

population-

based survey,

2003
24,284 Migrants based on nationalities from countries of birth: Afric Latin America, Asia, Balkan/Turkey, EU/USA; Angola, DR Congo, Morocco, Somalia, Tunisia, Brazil, Dominican Republic, Peru, Philippines, Sri Lanka, Thailand, Vietnam, Albania, Bosnia, Croatia, Kosovo, Macedonia, Serbia, Turkey, Austria, France, Germany, Italy, Netherlands, Poland, Portugal, Russia, Spain, UK, \& USA vs. 7500 Swiss women
Italy (Perugia)

Greece (Athens)

Italy (Rome)

Greece (Piraeus)

UK

Israel (Be'er Sheva)

US (San Diego)

Spain (Catalonia \& Valencia)

Italy (Bologna)

France
186 "Immigrant/foreign women" vs. 1716 Italian women

L overall (unadjusted)

181 "Immigrants" (majority from Albania, also included other Balkan countries and India/ Philippines/Pakistan) vs. 259 Greek women.

Only singleton births.

vs. vs. 11,976 Italian women.

1990 "Immigrants" (according to nationality) vs. 1081 Greek women.

Mediterranean, African (excluding East Africa), Bangladeshi, \& Pakistani vs. UK women.

431 Ethiopian Jewish immigrants vs. 20,047 Israeli Jewish women.

50 Hmong and Cambodian refugees vs. 25 Caucasian (non-Spanish, non-Oriental surnames).

34,746 Latin America, Eastern Europe, \& Maghreb vs. 180,633 Spanish women. Only singleton births.

510 Immigrant women from non-EU countries vs. 510 Western world (Italy, other EU, Australia, Canada, US). Only singleton births.

585 Africa (excluding North Africa), North Africa, Europe, \& "other" nationalities vs. 4658 French women. Only included low risk,

singleton births, with no previous caesarean

or medical indications for caesarean.

L overall (unadjusted) $\mathrm{H}$ during labour caesarean (unadjusted)

ND overall (unadjusted)

M overall (+ variation by parity; unclear if statistically significant)

ND overall (unadjusted)

L overall (unadjusted)

ND overall (unadjusted) ND emerg (unadjusted) L elect (unadjusted)

$H$ \& ND overall (not statistically tested) $H$ \& ND caesareans during labour (adjusted) $\mathrm{H}$ \& 
Table 1 Description of included studies (Continued)

\section{Rudman A, El-Khouri B, \& Waldenström U. National,}

(2008) ${ }^{\dagger}$ (English)

Rumbaut RG. (1996) (English)

Saurel-Cubizolles M-J, Saucedo M, Drewniak N, Blondel B, \& Bouvier-Colle M-H. (2012) (French)

Saurwein A. (1969) (German)

Schliemann F, \& Schliemann G. (1975) (German)

Schultze-Naumburg R, \& Scholtes G. (1976) (German, used English abstract)

Shah D, Tay A, Desai A, Parikh M, Nauta M, \& Yoong W. (2011) (English)

population-

based survey,

2000

Local, hospital records, 19891991

National, Populationbased survey 2010

Local, hospital records, 1964 1968

Local, populationbased registry 1969-1973

Local, hospital records, 19681973

Local, hospital records, 20062008

Shah RR, Ray JG, Taback N, Meffe F, \& Glazier R. (2011) (English)

Local, hospital records, 2002

Shah RR. (2007) (English) Thesis 2006

Sletten K. (2011) (English) Thesis unpublished ocal, hospital records, 2009201
Sweden

36 Swedish-speaking Foreign-born vs. 247 Swedish women.

ND overall (unadjusted) ND emerg

US (San Diego)

211 "Foreign-born \& ethnicity": defined as White, Asian, Hispanic, \& Black; Europe/

Canada, Middle East, Indo-Chinese, East Asian, Mexican, Central America \& Sub-Saharan Africa vs. 253 US born.

France

1864 Foreign-born (based on nationality) who could complete the survey in French:

European (including Turkey), North-African, Sub-Saharan African, \& other; $13 \%$ arrived in 2009/2010 and 36\% between 2005-2008. vs. 12,125 French women.

Germany (Cologne)

297 "Foreigners" from Southern Europe (including Turks, Greeks, Italian, \& Spanish women) vs. 7465 German women.

Germany (Hamburg)

217 "Foreigners/Guest workers" from Spain Italy, Portugal, Yugoslavia, Greece, Turkey \& "others" vs. 5112 German women.

Germany (Berlin)

UK (North London)

1941 "Foreign women" vs. 9009 German women.

125 First generation Chinese immigrant women (born in China). Economic migrants most likely and mean length of time in UK $=3.2$ years. vs. 125 British Caucasian women matched for age and parity.

Canada (Toronto) 3672 "Foreign born" based on country and region of birth using World Bank classification: Latin America/Caribbean, Western Europe/ USA/Japan/Australia/New Zealand, Eastern Europe/Central Asia, Middle East/North Africa, Sub-Saharan Africa, South Asia, East Asia/ Pacific. Asylum seekers examined based on health insurance. $<5$ years $\& \geq 5$ years also examined. Women with multiple gestation removed. vs. 1435 Canadian-born.

Norway (Baerum)

803 Immigrants from Latin America, Africa, Asia, Eastern Europe, \& Western Europe vs. 1634 Norwegian women. Low risk women $(35$ weeks or greater gestational age, no diabetes, singleton births) only. 
Table 1 Description of included studies (Continued)

\begin{tabular}{|c|c|c|c|c|c|}
\hline $\begin{array}{l}\text { Small R, \& Lumley J. (2007) }{ }^{\dagger} \text { (English) } \\
\text { Conference abstract }\end{array}$ & $\begin{array}{l}\text { Regional, } \\
\text { population- } \\
\text { based registry, } \\
\text { 1999-2007 }\end{array}$ & Australia (Victoria) & $\begin{array}{l}70,417 \text { Women from Non-English-speaking } \\
\text { countries (only countries w }>1000 \text { births were } \\
\text { included): Vietnam, China, Former Yugoslavia, } \\
\text { Philippines, Lebanon, India, Sri Lanka, Somalia, } \\
\text { Ethiopia, Eritrea, Sudan, Turkey, Malaysia, Iraq, } \\
\text { Cambodia, Indonesia, Greece, Germany, } \\
\text { Poland and Italy vs. 444, } 175 \text { Australian-born. }\end{array}$ & $\begin{array}{l}\text { M overall (+ variation by parity; not } \\
\text { statistically tested) }\end{array}$ & Fair \\
\hline $\begin{array}{l}\text { Small R, Gagnon A, Gissler M, et al. (2008) } \\
\text { (English) }\end{array}$ & $\begin{array}{l}\text { Regional \& } \\
\text { National, } \\
\text { population- } \\
\text { based registries, } \\
1997-2004\end{array}$ & $\begin{array}{l}\text { Australia (New South } \\
\text { Wales, Victoria), Belgium } \\
\text { (Flanders, Brussels), Canada } \\
\text { (Ontario, Quebec), Finland, } \\
\text { Norway, Sweden }\end{array}$ & $\begin{array}{l}\text { 10,431Somali women vs. } 2,168,891 \text { receiving- } \\
\text { country-born women. }\end{array}$ & $\begin{array}{l}\text { H overall [Belgium- Flanders (primips \& } \\
\text { multips) \& Belgium -Brussels]; H (primips) \& } \\
\text { ND (multips) overall (Australia- Victoria, } \\
\text { Finland, Norway, \& Sweden); ND overall } \\
\text { [Australia -New South Wales (primips \& } \\
\text { multips) \& Canada] (unadjusted) }\end{array}$ & Fair \\
\hline $\begin{array}{l}\text { Stray-Pedersen B, \& Austveg B. (1996) } \\
\text { (Norwegian) }\end{array}$ & $\begin{array}{l}\text { Local, hospital } \\
\text { records, } 1993\end{array}$ & Norway (Oslo) & $\begin{array}{l}734 \text { "Immigrants" (including Asia/Africa/South } \\
\text { America/East Europe and Turkey) vs. } 3188 \\
\text { Norwegians. }\end{array}$ & $\begin{array}{l}\mathrm{H} \text { overall (all immigrants combined; } \\
\text { unadjusted) }\end{array}$ & Fair \\
\hline $\begin{array}{l}\text { Teixeira C, Correia S, \& Barros H. (2010) } \\
\text { (English) Conference abstract }\end{array}$ & $\begin{array}{l}\text { Geographical } \\
\text { coverage not } \\
\text { indicated, } \\
\text { research study, } \\
\text { years not } \\
\text { indicated }\end{array}$ & Portugal & $\begin{array}{l}743 \text { European/North American, African, \& } \\
\text { South American (based on country of birth) } \\
\text { vs. } 6692 \text { Portuguese-born. Singleton births } \\
\text { only. }\end{array}$ & $\begin{array}{l}\text { H \& ND overall (adjusted but variables } \\
\text { included not indicated) }\end{array}$ & Fair \\
\hline $\begin{array}{l}\text { Triantafyllidis G, Tziouva K, Papastefanou I, } \\
\text { Samolis S, Katsetos C, \& Panagopoulos P. } \\
\text { (2010) (English) Conference abstract }\end{array}$ & $\begin{array}{l}\text { Local, hospital } \\
\text { records, } 2007\end{array}$ & Greece (Pireaus) & $\begin{array}{l}657 \text { "Immigrants" (based on nationality) } \\
\text { (mostly Albanians) vs. } 304 \text { Greek women. Only } \\
\text { term deliveries (gestational age 37-40 weeks). }\end{array}$ & ND overall (unadjusted) & Fair \\
\hline $\begin{array}{l}\text { Van Enk A, Doornbos HP, \& Nordbeck HJ. } \\
\text { (1990) (English) }\end{array}$ & $\begin{array}{l}\text { Local, hospital } \\
\text { records, 1972- } \\
1982\end{array}$ & Holland (Amsterdam) & $\begin{array}{l}1614 \text { Non-European immigrants defined by } \\
\text { ethnic origin: "Blacks" (Surinam \& Dutch } \\
\text { Antilles), Mediterranean (Turks \& Moroccans), } \\
\text { Asians [West Indian Asians (Hindustani from } \\
\text { Surinam), Chinese \& some Indonesians] vs. } \\
6234 \text { Dutch Caucasian. }\end{array}$ & $\begin{array}{l}\text { H \& ND overall (unadjusted; results consistent } \\
\text { when stratified by parity) }\end{array}$ & Fair \\
\hline $\begin{array}{l}\text { Van Enk WJ, Gorissen WH, \& Van Enk A. } \\
\text { (2000) (English) }\end{array}$ & $\begin{array}{l}\text { National, } \\
\text { population- } \\
\text { based registry, } \\
\text { 1990-1993 }\end{array}$ & Holland & $\begin{array}{l}5841 \text { Migrant teenagers (15-19 years old) } \\
\text { defined by ethnic and geographical } \\
\text { background: Mediterranean [Turkish \& North } \\
\text { African (mainly Moroccan)], Black (Surinam \& } \\
\text { Dutch Antilles), Hindustani (West Indian-Asian } \\
\text { from Surinam \& Dutch Antilles), Asian } \\
\text { (Chinese, Malaysian \& Malaccan); non-Dutch } \\
\text { European (West \& East Europe \& American), \& } \\
\text { "others" (mixed, unknown or other ethnicity) } \\
\text { vs. } 45,570 \text { Dutch (born in Netherlands and } \\
\text { West European origin, includes teenagers and } \\
20-24 \text { yr olds). Singleton, primiparous } \\
\text { pregnancies. }\end{array}$ & $\begin{array}{l}\text { M overall (when compared to Dutch teens); } L \\
\& \text { ND overall (when compared to } 20-24 \text { yr old } \\
\text { Dutch); (unadjusted but restricted to primips } \\
\text { and defined age groups) }\end{array}$ & Fair \\
\hline
\end{tabular}


Table 1 Description of included studies (Continued)

S, Stoltenberg C, \& Schei B. (1996)

(English)

Vangen S, Stoltenberg C, Skrondal A, Magnus

P, \& Stray-Pedersen B. (2000) (English)

records, 1992

National,

population-

based registry,

1986-1995

Vangen S, Stoltenberg C, Johansen RE,

National,

Sundby J, \& Stray-Pedersen B. (2002) (English) population-

based registry

1986-1998

Vangen S, Stray-Pedersen B, Skrondal A

Magnus P. \& Stoltenberg C. (2003) (English)

National

population-

based

registry, 1986-

1998

Vangen S, Stoltenberg C, Holan S, et al.

(2003) (English)

National

population-

Versi E, Liu KL, Chia P, \& Seddon G. (1995) (English)

ocal, hospital records, 1987199

Von Katterfeld B, Li J, McNamara B, \& Langridge AT. (2011) (English)

Regional populationbased registry 1998-2006

Local, hospital records, 2008

Walsh J, Robson M, \& Foley
(English) Conference abstract

Walsh J, Mahony R, Armstrong F, Ryan G, O'Herlihy C, \& Foley M. (2011) (English)

Local, hospital records, 2008
Norway

Norway

Norway

Norway 10,908 "Immigrants" (some has in North Africa (Morocco, Algeria and Tunisia), South Asia (Pakistan, Sri Lanka, India/Bangladesh) vs. 601,785 Norwegian women. Women with and without diabetes included.

UK (East London)

460 Bangladeshi vs. 7592 low-income Caucasian women

Australia (West Australia)

59,245 Foreign-born women as per mother's country of birth as declared in the birth register, ten regional categories as per Standard Australia Classification of countries: Oceania, North/West Europe, South/East

Europe, North Africa/Middle East, Sub Saharan Africa, South East Asia, North East Asia, South/ Central Asia, Americas vs. 149,737Australianborn (non-indigenous).

931 Eastern Europe, Africa, Britain, India, China M caesarean during labour (unclear if vs. 2499 Irish women. Only primiparous women, and delivered singleton, term infants.

552 Eastern European vs. 2449 Irish women. Primiparous, singleton, term deliveries for women who laboured.
ND overall (unadjusted) ND emerg

(unadjusted) ND elect (unadjusted)

overall (adjusted) H \& ND emerg (not

(not statistically tested)

H overall (adjusted) H emerg (adjusted) L elect (not statistically tested)

H overall (adjusted) $\mathrm{H}$ emerg (not statistically Good tested) H \& ND elect (adjusted)

ND overall (all immigrants combined; not

overall (alled ND emerg (all immigrants combined; not statistically tested) ND elect (all immigrants combined; not statistically tested)

ND overall (unadjusted; results consistent when stratified by parity) ND emerg (not statistically tested) $H$ (multips) \& ND (primips) emerg (unadjusted) L (primips \& multips) elect (unadjusted)

M overall (not statistically tested) H \& ND emerg (unadjusted) M elect (unadjusted) statistically significant)

ND caesarean during labourb (adjusted) ND caesarean pre-labour (excluded group from study)

Ryan G, Armstrong F, Walsh J \& Foley M.

(2010) (English) Conference abstract

Walsh J, Mahony R, McAuliffe F, O'Herlihy C,

Robson M, \& Foley M. (2009) (English)

Conference abstrac 
Table 1 Description of included studies (Continued)

Yoong W, Wagley A, Fong C, Chukwuma C, \& Local, hospital Nauta M. (2004) (English)

Yoong W, Kolhe S, Karoshi M, Ullah M, \& Nauta M. (2005) (English)

Zanconato G, Lacovella C, Parazzini F,

Local, research study, 2002

Local, hospital

Zlot Al, Jackson DJ, \& Korenbrot C. (2005) (English)

Zuppa AA, Orchi C, Calabrese V, et al. (2010) Local, research (English) Bergamini V, \& Franchi M. (2011) (English) records, 2002

2009

Local, hospital records, 1994-

1998

UK (North London)

UK (North London)

Italy (Verona) study, 2005

US (San Diego)

Italy (Rome)
61 Kosovo-Albanian vs. 61 British Caucasian.

ND overall (unadjusted)

Fair

69 Somali vs. 69 British Caucasian.

ND overall (unadjusted)

Fair

2661 "Immigrants" defined as 5 ethnic minority groups based on geographica D caesarean during labour (adjusted for age Europe, Middle East \& North Africa, Centra and South America, South and East Asia vs. 6365 Italian women; singleton births only.

1789 Mexican-born (categorized into acculturation groups by language abilityEnglish, Spanish and bilingual) vs. 313 US

born Latina women. Only includes low-risk, low income women.

585 "Immigrants" (includes women from Latin L overall (all immigrants combined; America, East Europe, West Europe, Russia, unadjusted) North America, Asia, Africa) vs, 2334 Italian-

* For "overall" "emerg" and "elect" caesarean rates for different migrant groups (defined by country/region of birth migration status, ethnicity, length of time in new country \&/or language ability) compared to nonmigrants: $\mathrm{H}=$ Higher rate(s); $\mathrm{L}=$ Lower rate(s); $\mathrm{ND}=$ No Different rate(s); $\mathrm{M}=$ Mixed (Higher \& Lower rates or Higher, Lower \& No Different rates); 'adjusted' if at minimum controlled for parity and age. Presented by migrants: $\mathrm{H}=$ Higher rate(s); $L=L$ Lower rate $(s) ; N D=N o$ Different rate(s); $M=$ Mixed (Higher $\& L O$

t Additional data provided. 
Table 2 Summary of study characteristics

Characteristic
Language of publication
English
French
Italian
Spanish
Norwegian
German

\section{Publication period}

$$
<1980
$$

1980-1989

1990-1999

2000-2012

Unpublished

Data Sources

Population-based data registries
Population-based hospital data
Population-based surveys
Research study/hospital records
ariables adjusted
Maternal age
Parity
Education
Gestational age
Marital status
Prenatal care
Social economic status (poverty, income,
under-housing, occupation)
Birthweight
Medical complications
Mechanical factors
Previous caesarean
Pregnancy complications (multiple, preterm,
fetal stress)
Smoking \& substance abuse
Medical indications
Onset of labour
Maternal weight/BMl/diabetes
Assisted reproductive technology
Infant sex
Hospital characteristics (level of care, size of
hospital, private facility)
Insurance status
Birth year
Tim

\begin{tabular}{ll}
$\begin{array}{l}\text { n (number } \\
\text { of studies) }\end{array}$ & $\%$ \\
\hline ( $=76)$ & \\
62 & 81.6 \\
4 & 5.3 \\
3 & 5.3 \\
1 & 2.6 \\
2 & 3.9 \\
4 & 1.3
\end{tabular}

$(n=76)$

13

45

$(n=76)$

21

2

49

$(n=19)^{*}$

19

17

7

3

4

9

Table 2 Summary of study characteristics (Continued)

Location of institution
Coverage
National
Regional
Local
Data years
$<1980$
1980-1989
1990-1999
$2000-2010$
Number of migrants studied
$<1,000$
$1,000-5,000$
$>5,000-10,000$
$>10,000$

\section{Source regions}

Sub-Saharan Africa

North Africa/West Asia

$(n=75)^{\dagger}$

15

19.7

$12 \quad 15.8$

$49 \quad 64.5$

$(\mathrm{n}=75)^{\ddagger}$

$12 \quad 12.6$

$17 \quad 17.9$

$28 \quad 29.5$

$38 \quad 40.0$

$(n=74)^{\S}$

$32 \quad 43.2$

$23 \quad 31.2$

$5 \quad 6.8$

$14^{* *} \quad 18.9$

$(n=1,029,454)^{+\dagger}$

$66,163 \quad 6.4$

$65,689 \quad 6.4$

$404,729 \quad 39.3$

$8855 \quad 0.9$

$68,084 \quad 6.6$

$69,589 \quad 6.8$

$70,545 \quad 6.9$

$46,842 \quad 4.6$

$18,005 \quad 1.7$

$27,922 \quad 2.7$

$58,065 \quad 5.6$

$16,336 \quad 1.6$

$108,630 \quad 10.6$

$(\mathrm{n}=76)^{\ddagger \neq}$

Receiving countries

11.1

Australia

Belgium 2

Canada 5

Finland $\quad 2 \quad 2.5$

$\begin{array}{lll}\text { France } & 6 & 7.4\end{array}$

Germany $\quad 4 \quad 4.9$

Greece $\quad 3 \quad 3.7$

Holland $\quad 2 \quad 2.5$

Ireland 3.7

Israel $\quad 3 \quad 3.7$

Italy $\quad 9 \quad 11.1$

Norway $\quad 10 \quad 12.3$

$\begin{array}{lll}\text { Portugal } & 1 & 1.2\end{array}$

$\begin{array}{lll}\text { Spain } & 3 & 3.7\end{array}$

$\begin{array}{lll}\text { Sweden } & 3 & 3.7\end{array}$ 
Table 2 Summary of study characteristics (Continued)

\begin{tabular}{|c|c|c|}
\hline Switzerland & 1 & 1.2 \\
\hline UK & 6 & 7.4 \\
\hline US & 9 & 11.1 \\
\hline Quality of reports & \multicolumn{2}{|c|}{$(n=76)$} \\
\hline Good & 22 & 28.9 \\
\hline Fair & 54 & 71.1 \\
\hline Caesareans & \multicolumn{2}{|c|}{$(n=64)^{\S \S}$} \\
\hline Higher & 13 & 20.3 \\
\hline Higher/No different & 12 & 18.8 \\
\hline Lower & 9 & 14.1 \\
\hline Lower/No different & 2 & 3.1 \\
\hline Mixed & 8 & 12.5 \\
\hline No different & 20 & 31.3 \\
\hline Emergency caesareans & \multicolumn{2}{|c|}{$(n=21)^{* * *}$} \\
\hline Higher & 7 & 33.3 \\
\hline Higher/No different & 4 & 19.0 \\
\hline Lower & 0 & 0.0 \\
\hline Lower/No different & 0 & 0.0 \\
\hline Mixed & 0 & 0.0 \\
\hline No different & 10 & 47.6 \\
\hline Elective caesareans & \multicolumn{2}{|c|}{$(n=19)^{* * *}$} \\
\hline Higher & 2 & 10.5 \\
\hline Higher/No different & 3 & 15.8 \\
\hline Lower & 5 & 26.3 \\
\hline Lower/No different & 2 & 10.5 \\
\hline Mixed & 1 & 5.3 \\
\hline No different & 6 & 31.6 \\
\hline \multicolumn{3}{|c|}{ 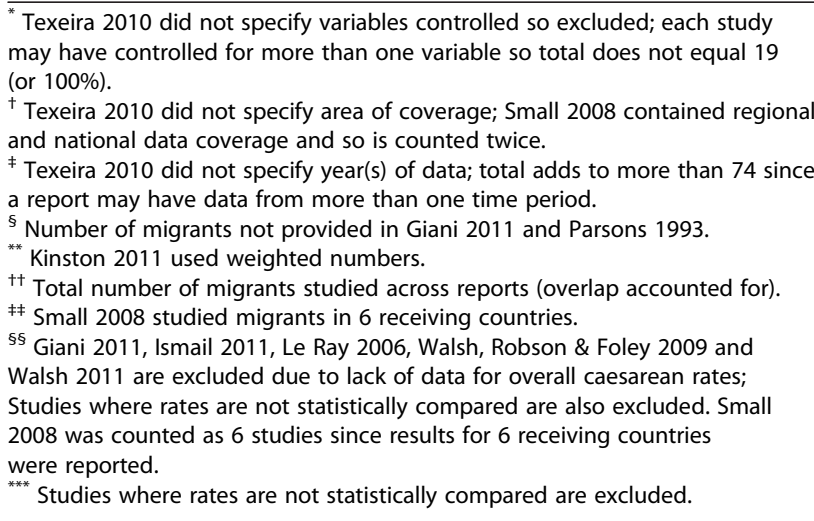 } \\
\hline
\end{tabular}

95\% CI $=1.92,2.58)][17,31,94]$, Australia [OR=1.17 (95\% $\mathrm{CI}=1.11,1.24)][34,91]$; Canada [OR=1.34 (95\% CI=1.08,1.67)] $[64,95]$ and North/West Europe [OR=1.43 (95\% CI=1.16, 1.77)] $[16,46,82]$. US $[37,57,81]$ and Southern European $[25,26,88]$ studies were too heterogeneous to combine, but tended towards higher rates. Analysis by parity showed primiparous African women to be more likely to have a caesarean $[\mathrm{OR}=2.24(95 \% \mathrm{CI}=1.63,3.08)]$
$[31,33,46,64]$ than non-migrants see Figure 1 ; multiparous women were also at increased risk $[\mathrm{OR}=2.02(95 \%$ $\mathrm{CI}=1.51,2.71)][31,46]$.

Analysis by type of caesarean suggests an excess risk of emergency caesareans for Sub-Saharan African women (all estimates above 1), although results were heterogeneous $[17,31,33,64,82,88,91,94]$. Risks for elective caesareans varied by receiving-country; three studies in France $[17,31,94]$, one in Italy [88] and another in Australia [91] showed higher risks of a planned caesarean, while two other studies, one in Canada [64] and the other in Norway [82], found African women to have the same and lower risk respectively.

Somali women in North America and Australia were found to have higher risks for a caesarean compared to non-migrant women $[\mathrm{OR}=1.13(95 \% \mathrm{CI}=1.02,1.26)]$ $[32,45]$. Significant heterogeneity prevented the calculation of a summary estimate for Somali women in Europe, though the tendency appeared similar to North America/Australia results.

Sub-analysis by parity shows primiparous Somali women to have greater risk for caesarean $[\mathrm{OR}=1.45$ (95\% CI $=1.30,1.62)][32,45]$ (see Figure 2). Multiparous Somali women also appear at increased risk however there was considerable heterogeneity preventing calculation of a summary estimate $[32,45]$. There were insufficient data for meta-analysis by type of caesarean in Somali women.

\section{North Africa (Maghreb)/West Asia (Middle East) (most studies reported results for North-Africa and West Asia combined)}

Sub-group analyses by receiving-country showed lower risk of a caesarean in Canada [OR=0.81 (95\% CI $=0.74,0.90)]$ $[64,97]$ and similar risk in France: [OR=1.09 (95\% CI=0.95, 1.26)] $[17,31,56,73,94]$. Analysis for other countries/regions yielded heterogeneous results. With respect to type of caesarean, North African/West Asian migrant women had an elevated risk if the caesarean was an emergency [OR $=1.11$ $(95 \% \mathrm{CI}=1.03,1.20)][15,17,31,33,64,88,91,94]$.

\section{Latin America}

Differences in caesarean rates between Latin American migrants and non-migrant women depended on receivingcountry. Rates were higher in Norway [OR $=2.41(95 \%$ $\mathrm{CI}=1.79,3.23)][15,82]$, and Canada $[\mathrm{OR}=1.43(95 \% \mathrm{CI}=1.29$, 1.59)] [64,97], whereas in Southern Europe rates were similar $[\mathrm{OR}=1.03(95 \% \mathrm{CI}=0.94,1.12)][26,53,88]$. Results in other receiving countries were too few or heterogeneous to combine. Latin American women showed greater risk for an emergency caesarean compared to non-migrant women $[\mathrm{OR}=1.59(95 \% \mathrm{CI}=1.13,2.25)][15,64,82,88]$. Results for elective caesareans were too heterogeneous for calculation of a summary estimate. 


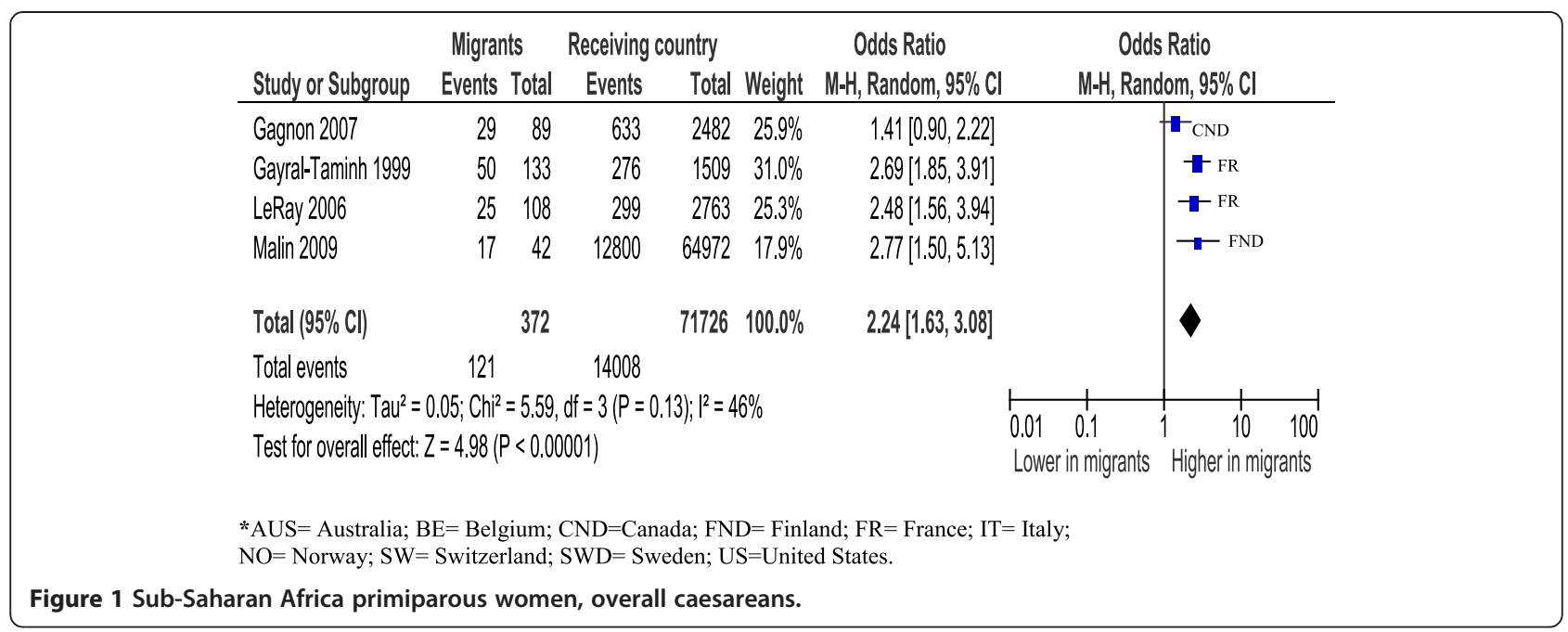

\section{Caribbean (non-Hispanic)}

Three older European studies provided data for migrants from former colonized Caribbean states (Holland, UK, France) and combined showed these women were more likely to have caesarean than non-migrants $[\mathrm{OR}=1.91$ (95\% CI=1.37, 2.66)] [72,73,78].

\section{South Asia}

Combining studies with similar populations (India, Sri Lanka, unspecified) show these migrant women have an excess of caesareans compared to receiving-country-born women $[\mathrm{OR}=1.28$ (95\% CI=1.22, 1.35)] (see Figure 3) $[15,16,26,46,58,61,97]$. Examined by parity, both primiparous and multiparous women had more caesareans $[\mathrm{OR}=1.19(95 \% \mathrm{CI}=1.12,1.25)$ and $\mathrm{OR}=1.39(95 \% \mathrm{CI}=1.31$, $1.47)$ respectively] $[46,61]$. Studies for emergency caesareans were too heterogeneous, and there were insufficient data to examine elective caesareans.

\section{East Asia ("Far East")}

East Asian women in Southern Europe and the US are less likely to have a caesarean:[OR=0.59, (95\% CI=0.47, 0.73)] $[25,26]$ and $[\mathrm{OR}=0.73(95 \% \mathrm{CI}=0.71,0.75)][37,81]$ respectively, whereas East Asian women in Australia, the UK, Canada and Finland had similar rates to non-migrant women $[\mathrm{OR}=0.99(95 \% \mathrm{CI}=0.95,1.03)][46,61,91,93,97]$.

Sub-analysis of three studies of Chinese women $[46,61,93]$ show no difference between migrant and non-migrant women overall $[\mathrm{OR}=0.97(95 \% \mathrm{CI}=0.75,1.25)]$, for primiparous women $[\mathrm{OR}=0.93(95 \% \mathrm{CI}=0.86,1.00)]$ or for multiparous women $[\mathrm{OR}=1.02(95 \% \mathrm{CI}=0.95,1.10)][46,61]$.

\section{South-East Asia}

Despite significant heterogeneity, migrant women from Vietnam, Thailand, Cambodia, and Laos had lower risks of caesarean compared to non-migrant women in all nine included studies $[15,16,37,46,48,49,51,60,61]$. Focusing on

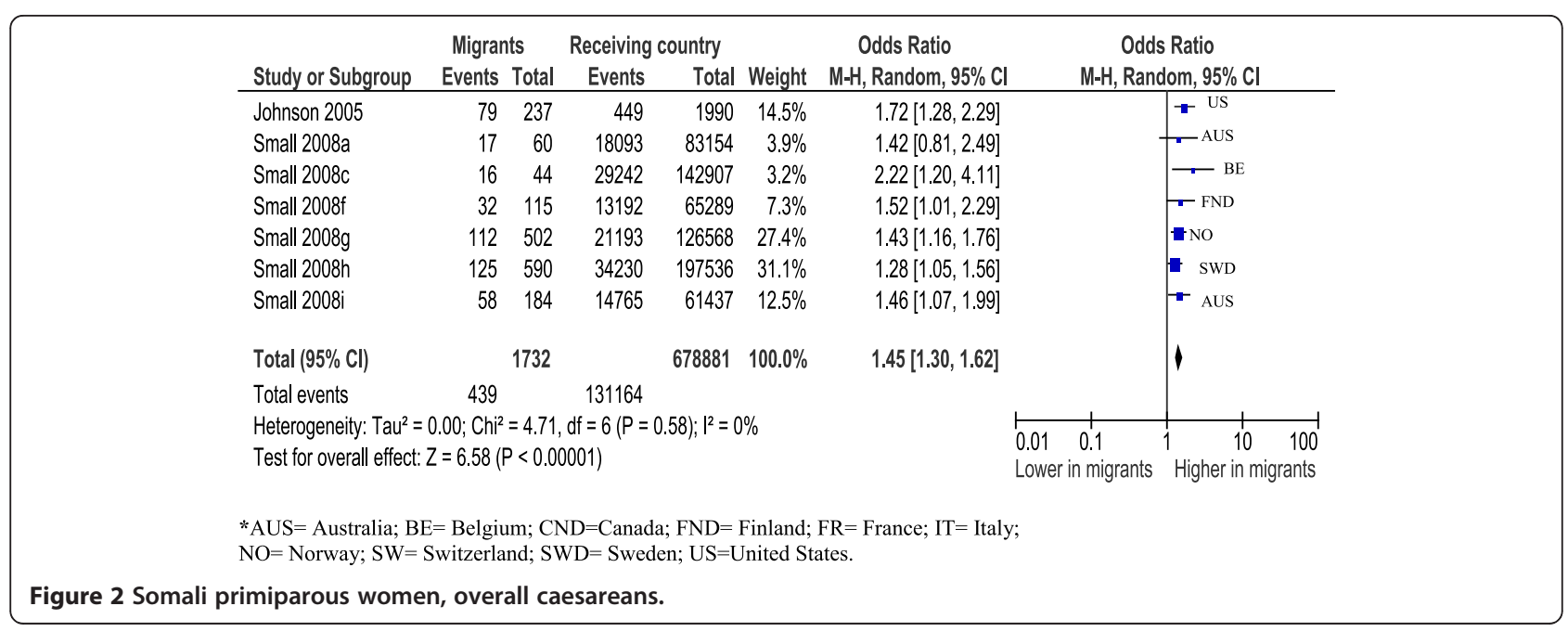


Vietnamese women, two older studies found Vietnamese to have caesarean rates lower than Australian-born [OR= 0.87 (95\% CI $=0.75,1.00][49,60]$. Similarly more recent studies showed Vietnamese women to have lower rates compared to non-migrant women $[\mathrm{OR}=0.68$ (95\% CI=0.66, $0.71)][16,46,61]$. Results were consistent for primiparous $[\mathrm{OR}=0.78(95 \% \mathrm{CI}=0.64,0.94)]$ and multiparous $[\mathrm{OR}=0.65$ (95\% CI=0.62, 0.68)] women $[46,49,61]$.

Contrary to other South East Asian women, Filipino women tended towards a greater risk for caesarean birth, although heterogeneity prevented calculation of a summary point estimate $[15,16,28,39,49,52,61]$. Two Australian studies show multiparous Filipino women to have higher rates [OR=1.19 (95\% CI=1.10,1.29)] [49,61].

\section{Eastern Europe}

Results of studies reporting comparisons of Eastern European women to receiving-country-born are too heterogeneous to calculate a summary point estimate but suggest these women have lower risks for caesarean, with all estimates lower or tending to be lower than one $[26,46,53,82,88,97]$. Analysis specifically of primiparous women confirmed a reduced risk $[\mathrm{OR}=0.52, \quad(95 \%$ $\mathrm{CI}=0.43,0.63)][46,87,96]$. A sub-analysis of Kosovo women $[\mathrm{OR}=0.49,(95 \% \mathrm{CI}=0.36,0.67)][16,43]$ and women from Russia/Baltic States living in Europe (Switzerland and Finland) also showed these women to have lower risks $[\mathrm{OR}=0.75,(95 \% \mathrm{CI}=0.66,0.85)][16,46]$.

\section{Southern Europe}

Comparisons of women from Italy, Portugal, Spain, and Greece migrating to other parts of Europe or Australia were heterogeneous although the risks of caesarean were consistently greater than that of receiving-country-born women $[16,52,56,61,63,68,73]$.

\section{Other}

Analyses by other migration indicators (migration status and length of time in receiving-country) were possible for migrants in Canada only. These included an analysis of asylum-seekers who showed no difference in rates compared to Canadian-born $[\mathrm{OR}=0.93(95 \% \mathrm{CI}=0.74,1.17)]$ $[64,66,95]$; and migrant women in Canada five years or less who showed a greater risk for caesarean $[\mathrm{OR}=1.14(95 \%$ $\mathrm{CI}=1.06,1.23)][64,66,97]$.

\section{Sensitivity analyses}

Meta-analyses with population-based studies confirmed the findings with consistent results for Sub-Saharan African women in France $[\mathrm{OR}=2.19$ (95\% CI=1.80,2.67)] $[17,94]$, and South Asian women $[\mathrm{OR}=1.25$ (95\% CI=1.17, $1.33)][16,26,46,61,97]$. Sensitivity analyses also support the findings of higher risk of emergency caesareans for North-African/West Asian women $[\mathrm{OR}=1.09$ (95\% CI= $1.00,1.19)][15,17,91,94]$ and lower risk of caesareans for Eastern European women (heterogeneous but similar estimate). Sensitivity analyses were not informative for Somali and Vietnamese migrants since all studies initially meta-analyzed were population-based, and for Latin American migrants due to an insufficient number of studies.

Summary estimates of adjusted ORs for overall caesarean rates for Sub-Saharan African $[\mathrm{OR}=1.41 \quad(95 \%$ $\mathrm{CI}=1.19,1.66)] \quad[16,65,88,95]$ and Somali women $[\mathrm{OR}=1.99(95 \% \mathrm{CI}=1.44,2.75)][16,32,41]$ were consistent in showing higher rates of caesarean compared to non-migrant women. Inconsistent findings were found however when adjusted meta-analyses were performed for South Asian and Latin American migrants; South Asian women still tended towards higher rates but with a wide, non-significant confidence interval $[\mathrm{OR}=1.10$ $(95 \% \mathrm{CI}=0.87,1.38)][16,95]$ and Latin American women 
showed no difference in emergency caesarean rates compared to non-migrant women $[\mathrm{OR}=1.01 \quad(95 \% \mathrm{CI}=0.62$, 1.63)] $[82,88]$. Adjusted analyses for emergency caesareans in North-African/West Asian women showed a similar point estimate to the unadjusted analyses but with a wide, nonsignificant confidence interval $[\mathrm{OR}=1.16(95 \% \mathrm{CI}=0.86,1.56)]$ $[17,88]$. Adjusted results for Eastern European women were too heterogeneous, but did generate a similar estimate to the unadjusted results.

\section{Assessment for publication bias}

Visual inspection of the individual funnel plots for studies reporting overall caesarean rates for Sub Saharan African, Somali, South Asian, Eastern European, Vietnamese, Latin American and North African/West Asian migrants (and emergency caesareans for the two latter) all showed symmetry, suggesting no publication bias, although very small studies were generally lacking.

\section{Mechanisms/indications for caesareans among migrant women}

Table 3 summarizes important risk factors/mechanisms for caesareans in migrants cited in included studies and for each factor the number of studies citing this factor/ mechanism. Combined the most commonly reported (in order of frequency) were: language/communication barriers, low social economic status (SES), poor maternal health (e.g., anaemia, STIs, TB, parasitic infections), gestational diabetes/high body mass index (BMI), fetopelvic disproportion, and lack of prenatal care.

Evidence to explain the consistently different rates of caesarean between Sub Saharan African, Somali, South Asian, Vietnamese, Eastern European, Latin American and North African/West Asian (emergency caesareans for the two latter) and non-migrant women, was limited. Variables adjusted (see Table 2) or stratified differed across studies and few studies compared indications for caesarean between migrant and non-migrant groups making it difficult to draw firm conclusions regarding explanatory risk factors or mechanisms.

For Somali [15,16,32,41] and Sub-Saharan African $[16,17,82,88,95]$ migrants, adjusted analyses indicate factors other than maternal age, parity, birthweight, or medical complications [e.g., preterm, feto-pelvic disproportion, hypertension, diabetes, BMI] are involved, although it is difficult to know which factors. Prolonged labour due to pelvic shape $[17,31,71]$, genital cutting $[15,32,41,45,46,57,88]$, language barriers $[15-17,32,45]$, poor maternal health (e.g., infectious diseases, anaemia) [31,46,65], a lack of prenatal care [16,33]' and low SES [16,31,32] have all been postulated to be important mechanisms for these women. Gestational diabetes mellitus (GDM) and dystocia were also mentioned as a concern for Sub-Saharan African/Somali women (more than receiving-country-born women) in some studies $[32,34,46]$.

Reports that provided comparisons of medical indications for caesareans between Sub-Saharan African/Somali and non-migrant women show these women to have more caesareans due to fetal distress [31,32] and failed induction [32]. Similarly one study found Sub-Saharan African women who were induced to be more likely to have a caesarean compared to French women who were induced [17], suggesting complications with labour that could be due to any number of different reasons.

Adjusted analyses for South Asian [15] and Latin American (emergency caesareans) [82,88] migrants show age, parity or medical complications (e.g., feto-pelvic disproportion, fetal distress) partially explain excess caesareans in these women, although it is not possible to isolate which of these factors is important. For Latin American women, studies which show consistently higher rates of caesarean irrespective of adjustment of covariates or stratification (e.g., birthweight, hospital type) suggest different contributing factors $[15,27,53,65,95]$. Seeking social status [55] or cultural preference $[15,46,53,95]$ is one suggested cause; higher rates of pre-eclampsia might also have a role [46]. Stratified analyses by neighbourhood (English-speaking and non-English-speaking) have shown higher rates of caesarean for Latinas compared to USborn when living in non-English-speaking neighbourhoods, leading to the suggestion that communication or cultural barriers might be explanatory mechanisms [27].

For North African/West Asian migrants, hypothesized mechanisms leading to caesarean birth included illness (e.g., hypertension, diabetes) [73], low SES resulting in reduced access to prenatal care $[33,73]$, language barriers [56], and macrosomia [29,70].

Vietnamese [15] and Eastern European [53,87,96] migrants seem to have protective factors that explain their consistently lower rates of caesarean. Proposed protective factors include a preference for a vaginal birth [15,87]; the healthy-immigrant effect (i.e., in which immigrants are healthier than the native population due to immigration selection criteria which excludes individuals with significant health problems) [87,93]; a healthier lifestyle (no smoking, drinking alcohol, or drug abuse; low BMI) $[87,96]$; young maternal age $[87,96]$; social support $[16]$ and the use of fewer interventions during labour and birth $[87,96]$. In Southern Europe (Italy, Spain, Portugal), lower rates among migrants are also thought to be due to a preference for caesareans among non-migrant women $[30,50]$ and because healthcare professionals are more concerned about litigation $[25,36,86]$ from non-migrants.

Filipino migrants were explicitly discussed in a number of studies and hypotheses for their higher caesarean rates included maternal preference $[15,39]$ and fetopelvic disproportion due to interracial marriage resulting 
Table 3 Potential mechanisms \& risk factors involved in caesareans among migrants

\begin{tabular}{ll}
\hline Mechanism/Risk factor & N \\
\hline Income and social status & 15 \\
Low SES (education, income) & 5 \\
Higher social status & 2 \\
No legal status & 1 \\
Poor nutritional status & \\
Social support networks & \\
No partner and/or family, friends & 3 \\
Education and literacy & \\
High education & \\
Employment and working conditions \\
Low status job \\
Social environment \\
Poor living conditions \\
Physical environment \\
Violence, trauma or experiences of abuse \\
Personal health practices \\
High BMl \&/or Gestational diabetes/diabetes \\
Smoking, drug or alcohol abuse \\
Healthy child development \\
Fetal distress \\
Low birthweight \\
Prematurity
\end{tabular}

Biology and genetic endowment

Illness and other pathologies (e.g., anaemia, hepatitis, TB, malaria, HIV/STIS, parasitic or other infections)

Pelvis shape/size (feto-pelvic disproportion)

Older age

Short stature

Pre-eclampsia/hypertension

Prolonged labour/failure to progress

Post-datism

Health services

Language/communication barriers

Lack of healthcare including prenatal care

Discrimination/racism

Hospital environment

Gender

Genital cutting

Culture

Beliefs/preferences about birth

Grand-multiparity

Inter-racial marriage (leading to large birthweight babies)
Table 3 Potential mechanisms \& risk factors involved in caesareans among migrants (Continued)

Acculturation (adoption of unhealthy lifestyles) 3

Different concepts of health and disease (leading to 2 different health seeking behaviour)

Reduced food intake during pregnancy (to have a 1 smaller fetus)

${ }^{*}$ Mechanisms/Risk Factors are organized under 12 determinants of population health (Public Health Agency of Canada (PHAC). Immigration and HealthExploring the Determinants of Health. Ottawa: Public Health Agency of Canada; 2011).

in large birthweight babies $[15,28,39,49,52]$. More caesareans due to dystocia and feto-pelvic disproportion have been shown, however, this was deemed to not be due to interracial marriage (by comparing Filipino women in mixed marriages to Filipino women in non-mixed marriages) $[28,39]$. Filipino women who gave birth by caesarean were also found to experience a greater number of medical and obstetric complications (e.g., GDM, anaemia, viral diseases) compared to Australian-born women who delivered by caesarean [28].

\section{Discussion}

Our review of 76 studies comparing the rates of caesarean births between migrant and non-migrant women living in OECD countries, show that women from SubSaharan Africa, Somalia and South Asia consistently have an excess of caesareans compared to receivingcountry-born women while Eastern European and Vietnamese women have lower overall caesarean rates. North African/West Asian and Latin American women have higher emergency caesarean rates.

The literature provides inadequate empirical evidence to explain differences in caesarean rates observed. Overall it appears that a combination of factors and mechanisms are likely to be involved. The most frequently postulated risk factors for caesarean risk in migrant populations include: language/communication barriers, low SES, poor maternal health, gestational diabetes/high BMI, feto-pelvic disproportion, and lack of prenatal care. There were no studies identified examining determinants of caesareans in migrants specifically. However, gestational diabetes mellitus (GDM) and feto-pelvic disproportion are known to complicate delivery and increase the risk of caesarean [104,105] and these are more common in some migrant women $[28,39,106]$. Reasons for increased risk of GDM in migrants are unknown but might be associated with a genetic predisposition, or physiological response to dietary changes; the involvement of a stress response has also been proposed. Feto-pelvic disproportion might be the result of short stature, a large birthweight baby or childhood malnutrition [107]. 
A BMI above normal is associated with a number of adverse reproductive outcomes, including GDM, macrosomia, prolonged labour, pre-eclampsia and caesarean $[108,109]$, although the exact mechanisms have not been identified. Excessive weight among childbearing women also varies by ethno-cultural background, with African women representing one of the groups with the highest rates of obesity $[94,110]$.

Inadequate prenatal care is more common among migrants [111]. Language and no health insurance are often cited barriers to healthcare [11,112]. Women who do not receive prenatal care, or who cannot communicate their medical history, may have undiagnosed and untreated medical conditions and they do not benefit from other preventative interventions (e.g., advice about prenatal nutrition) [113]. Medical issues that may not be identified include parasitic infections, anaemia and TB which are known to affect some childbearing migrant women [114-116], although their association with caesarean birth is unclear.

Poverty, unemployment and low social status are significant concerns for migrants $[117,118]$. Low SES may indirectly be protective against caesarean since women of low SES are less likely to receive care in a private facility and there may be less concern of litigation among healthcare professionals caring for these women. However, low SES may be a barrier to accessing prenatal care, and is known to be independently associated with poorer health. Qualitative studies suggest that a lack of information and/or support during pregnancy and birth due to marginalization, communication barriers, and/or cultural insensitivity (e.g., genital cutting), may result in anxiety, fear and disempowerment $[119,120]$ and lead to unnecessary caesareans.

Confirmation and further understanding of social support as a protective mechanism could have positive implications for reducing caesarean rates among migrants. Additional support (in addition to routine care) including emotional support as well as practical assistance provided during pregnancy has been shown to reduce the risk of caesarean birth for women in socially disadvantaged situations [121]. Continuous support during labour is also associated with a reduced risk of caesarean [122]. This strategy may be particularly beneficial for reducing emergency caesareans, which overall appear to be more common among migrants.

Our findings also suggest that receiving-country is an important variable to consider, particularly for certain groups (e.g., North African, Latin American, East Asian). The degree to which variation in caesarean outcomes across countries represents effects of policies and/or healthcare delivery [123] or other particularities of receiving countries (e.g., cultural factors) versus differences in the migrant populations resettling in each country, remains unknown. More precise definitions of migrants and more complete individual level migration-related data, including source country, length of time in host country, receiving-country language ability (at the time of pregnancy and birth), and migration status (e.g., refugee or economic immigrant) [124] would allow for better interpretation of results particularly since results from Canadian studies have shown that length of residence post-migration ( $<5$ years) was a significant predictor of more caesarean births. Confirmation of these findings in other OECD countries would be informative.

Research to establish evidence for risk factors associated with caesarean birth in migrants and to deconstruct the pathways (e.g., genetic or physiological, psychological stress, delivery of maternity care) by which they lead to disparities in mode of birth outcomes, is needed. Pathways are likely multi-factorial and complex. Future work using a combination of quantitative and qualitative approaches may be valuable in more fully expounding the processes. Moreover, greater emphasis on differences in caesarean indications/ mechanisms would be more informative than simply comparing caesarean rates between migrant and non-migrant women since rates may not be higher, but there may still be disparities in risk factors for caesarean births.

\section{Strengths and limitations}

There are limitations to this review. The web searches, although extensive, did not include all of the government and professional agency websites from all OECD countries. The majority of included studies were rated as 'fair' quality for not controlling for confounding or due to some ambiguity in their definitions of the study groups. The heterogeneity due to variation in the migrant populations studied or how source countries were grouped to represent regions, made it challenging to combine data for metaanalysis. US studies were largely missing from metaanalyses due to heterogeneity (for Sub-Saharan African women) or lack of data for the source regions analyzed (for Eastern European, Vietnamese, South Asian, Latin American and North-African/West Asian women). This might be problematic since other systematic reviews comparing the perinatal health of migrants to non-migrants $[125,126]$ had varied outcomes between US and European regions. Nonetheless, a broad range of studies was included and analyzed; the database searches were exhaustive with no time or language limitations and only three reports could not be reviewed due to language. Rigorous methods for reviewing, extracting and analyzing data were applied, optimizing the quality of the results generated. The consistency of the results with population-based data offers confidence in the robustness of the findings.

A major strength of this review is that it is the first of which we are aware to systematically examine hypotheses put forward to explain differences in caesarean rates 
between migrant and non-migrant women and to assess these in light of the available empirical evidence.

\section{Conclusion}

Sub-Saharan African, Somali, and South Asian migrants consistently have higher caesarean rates while EasternEuropean and Vietnamese migrants have lower overall caesarean rates compared to receiving-country-born women. North-African/West Asian and Latin American migrant women have higher emergency caesarean rates. To date there is inadequate empirical evidence to explain observed differences in caesarean rates; more focused research is urgently needed.

\section{Additional files}

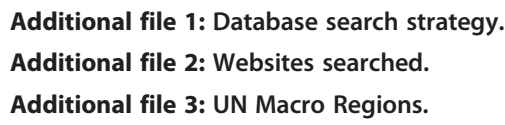

\section{Competing interests}

The authors declare that they have no competing interests.

\section{Authors' contributions}

LM reviewed the literature, extracted data, performed analyses and wrote the first draft of the paper. AG conceived the study, reviewed literature, and critically revised the manuscript for important intellectual content. RS and BB contributed to the design of the study and critically revised the manuscript for important intellectual content. All authors approved of the final version of the paper to be published.

\section{Acknowledgements}

The ROAM (Reproductive Outcomes and Migration) collaboration for locating relevant literature and providing assistance to review non-English reports. Luisa Ciofani (McGill University Health Centre \& Canadian Association of Perinatal and Women's Health Nurses (CAPWHN)); Vyta Senikas (Society of Obstetricians and Gynaecologists of Canada (SOGC)); and Emmanuelle Hébert (Canadian Association of Midwives (CAM) for their professional input to the manuscript.

\section{Funding}

Canadian Institutes of Health Research (CIHR) provided funds to support the research (FRN: 103265). The McGill University Health Centre- Research Institute provided research space and administrative support. Lisa Merry holds a FRSQ (Fonds de Recherche Santé Québec) Doctoral training award and a FRESIQ (Fondation de recherche en sciences infirmières du Québec) doctoral bursary through their MELS Universities' program and is also funded by the Strategic Training Program in Global Health Research, a partnership of the Canadian Institutes of Health Research and the Québec Population Health Research Network.

\footnotetext{
Author details

${ }^{1}$ Ingram School of Nursing, McGill University, Montreal, QC, Canada. ${ }^{2}$ Mother and Child Health Research, La Trobe University, Melbourne, VIC, Australia. ${ }^{3}$ Unité 953, Recherche épidémiologique en santé périnatale et santé des femmes et des enfants, Institut National de la Santé et de la Recherche Médicale (INSERM), Paris, France. ${ }^{4}$ Ingram School of Nursing and Department of Obstetrics and Gynecology, McGill University; McGill University Health Centre (MUHC), Montreal, QC, Canada.
}

Received: 4 October 2012 Accepted: 23 January 2013

Published: 30 January 2013

\section{References}

1. Betrán AP, Merialdi M, Lauer JA, Bing Shun W, Thomas J, Van Look P, Wagner M: Rates of caesarean section: analysis of global, regional and national estimates. Paediatr Perinat Epidemiol 2007, 21:98-113.

2. Deneux-Tharaux C, Carmona E, Bouvier-Colle MH, Bréart G: Postpartum maternal mortality and cesarean delivery. Obstet Gynecol 2006, 108:541-548.

3. World Health Organization: Appropriate technology for birth. Lancet 1985, 2:436-437.

4. United Nations, Department of Economic and Social Affairs, Population Division: Trends in International Migrant Stock: The 2008 Revision (United Nations database, POP/DB/MIG/Stock/Rev.2008). New York: United Nations; 2009. Available at: http://www.un.org/esa/population/publications/ 2009Migration_Chart/ittmig_wallchart09.pdf.

5. Statistics Canada: Canada's demographic situation: Fertility of immigrant women. The Daily 2003, Monday, December 22. Available at: http://www. statcan.gc.ca/daily-quotidien/031222/dq031222c-eng.htm.

6. Sobotka T: Overview Chapter 7: the rising importance of migrants for childbearing in Europe. Demographic Research 2008, 19:225-248.

7. World Health Organization: Health of Migrants -The Way Forward: report of a global consultation, Madrid, Spain, 3-5 March 2010. Geneva: World Health Organization Press; 2010. Available at: http://www.who.int/hac/events/ consultation_report_health_migrants_colour_web.pdf.

8. International Organization for Migration (IOM): International Migration Law Glossary on Migration. 2nd edition. Geneva: IOM; 2011.

9. United Nations High Commissioner for Refugees (UNHCR): Refugee Status Determination, Identifying who is a refugee. Geneva: UNHCR; 2005.

10. Fuentes-Afflick E, Hessol NA: Immigration status and use of health services among Latina women in the San Francisco Bay Area. J Womens Health 2009, 18:1275-1280.

11. terKuile S, Rousseau C, Munoz M, Nadeau L, Ouimet MJ: The universality of the Canadian health care system in question: Barriers to services for immigrants and refugees. Int J Migr Health Soc Care 2007, 3:15-26.

12. Eisenman DP, Keller AS, Kim G: Survivors of torture in a general medical setting: how often have patients been tortured, and how often is it missed? West J Med 2000, 172:301-304.

13. Carballo M, Nerukar A: Migration, refugees, and health risks. Emerg Infect Dis 2001, 7(3 Suppl):556-560.

14. Gagnon AJ, Zimbeck M, Zeitlin J: Migration to western industrialized countries and perinatal Health: A systematic review. Soc Sci Med 2009, 69:934-946

15. Vangen S, Stoltenberg C, Skrondal A, Magnus P, Stray-Pedersen B: Cesarean section among immigrants in Norway. Acta Obstet Gyn Scan 2000, 79:553-558.

16. Merten S, Wyss C, Ackermann-Liebrich U: Caesarean sections and breastfeeding initiation among migrants in Switzerland. Int J Public Health 2007, 52:210-222.

17. Roman H, Blondel B, Bréart G, Goffinet F: Do risk factors for elective cesarean section differ from those of cesarean section during labor in low risk pregnancies? J Perinat Med 2008, 36:297-305.

18. Stroup DF, Berlin JA, Morton SC, Olkin I, Williamson GD, Rennie D, Moher D, Becker BJ, Sipe TA, Thacker SB: Meta-analysis of observational studies in epidemiology: a proposal for reporting. Meta-analysis Of Observational Studies in Epidemiology (MOOSE) group. JAMA 2000, 283:2008-2012.

19. Harris RP, Helfand M, Woolf SH, Lohr KN, Mulrow CD, Teutsch SM, Atkins D, Methods Work Group, Third US Preventive Services: Current methods of the US Preventive Services Task Force: a review of the process. Am J Prev Med 2001, 20(3 Suppl):21-35.

20. Higgins JP, Green S (Eds): Cochrane Handbook for Systematic Reviews of Interventions, Version 5.1.0 [updated March 2011], The Cochrane Collaboration: 2011. Available at: http://handbook.cochrane.org/

21. Centre for Epidemiology and Research, NSW Department of Health: New south wales mothers and babies 2008. NSW Public Health Bull 2010, 21

22. van Enk W, Gorissen W, van Enk A: Tienerzwangerschappen naar etniciteit in Nederland, 1990-1993. Ned Tijdschr Geneeskd 1999, 143:465-471.

23. Herpola O: Indications for caesarean section. Duodecim 1947, 63:25-30.

24. Wittlinger H, Beck HO, Brandner U: Geburtshilfe Frauenheilkd 1971, 1174-1183.

25. Alonso CP, Maresca MI, Alonso OT, Moro SM: Perinatology of newborns of immigrant parents at Hospital Clinico 'San Carlos' in Madrid, Spain. Acta Pediatr Esp 2006, 64:61-67.

26. Bona G, Zaffaroni M, Cataldo F, Sandri F, Salvioli GP: Infants of immigrant parents in Italy. A national multicentre case control study. Panminerva Med 2001, 43:155-159. 
27. Braveman $P$, Egerter S, Edmonston F, Verdon M: Racial/ethnic differences in the likelihood of cesarean delivery, California. Am J Public Health 1995, 85:625-630

28. Cassell E: An Investigation of the Adverse Obstetric Profile and Pregnancy Outcomes Among Philippines-Born Women in Victoria, 1982-92. In Thesis. Monash University: Department of Social and Preventive Medicine; 1995

29. Delvaux T, Buekens P, Thoumsin H, Dramaix M, Collette J: Cord C-peptide and insulin-like growth factor-l, birth weight, and placenta weight among North African and Belgian neonates. Am J Obstet Gynecol 2003, 189:1779-1784.

30. Diani F, Zanconato G, Foschi F, Turinetto A, Franchi M: Management of the pregnant immigrant woman in the decade 1992-2001. J Obstet Gynaecol 2003, 23:615-617.

31. Gayral-Taminh M, Arnaud C, Parant O, Fournie A, Reme JM, Grandjean H: Pregnancy and labor of women born in Maghreb and Black Africa followed to delivery at the Maternity Hospital of Toulouse. J Gynecol Obstet Biol Reprod 1999, 28:462-471.

32. Johnson EB, Reed SD, Hitti J, Batra M: Increased risk of adverse pregnancy outcome among Somali immigrants in Washington state. Am J Obstet Gynecol 2005, 193:475-482.

33. LeRay C, Carayol M, Zeitlin J, Breart G, Goffinet F, PREMODA Study Group: Level of perinatal care of the maternity unit and rate of cesarean in lowrisk nulliparas. Obstet Gynecol 2006, 107:1269-1277.

34. Ma J, Bauman A: Obstetric profiles and pregnancy outcomes of immigrant women in New South Wales, 1990-1992. Aust N Z J Obstet Gynaecol 1996, 36:119-125.

35. Panagopoulos P, Tsoukalos G, Economou A, Zikopoulos M, Koutras I, Petrakos G, Pachakis M: Delivery and immigration: the experience of a Greek Hospital. Clin Exp Obstet Gynecol 2005, 32:55-57.

36. Rizzo N, Ciardelli V, Gandolfi-Colleoni G, Bonavita B, Parisio C, Farina A, Bovicelli L: Delivery and immigration: the experience of an Italian hospital. Eur J Obstet Gynecol Reprod Biol 2004, 116:170-172.

37. Rumbaut R: Unraveling a public health enigma: Why do immigrants experience superior perinatal health outcomes. Res Sociol Health Care 1996, 13B:337-391.

38. Vangen S, Stoltenberg C, Schei B: Ethnicity and use of obstetrical analgesia: do Pakistani women receive inadequate pain relief in labour? Ethn Health 1996, 1:161-167.

39. Vangen S, Stray-Pedersen B, Skrondal A, Magnus P, Stoltenberg C: High risk of cesarean section among ethnic Filipinos: an effect of the paternal contribution to birthweight? Acta Obstet Gynecol Scand 2003, 82:192-193.

40. Vangen S, Stoltenberg C, Holan S, Moe N, Magnus P, Harris JR, StrayPedersen B: Outcome of pregnancy among immigrant women with diabetes. Diabetes Care 2003, 26:327-332.

41. Vangen S, Stoltenberg C, Johansen RE, Sundby J, Stray-Pedersen B: Perinatal complications among ethnic Somalis in Norway. Acta Obstet Gynecol Scand 2002, 81:317-322.

42. Versi E, Liu KL, Chia P, Seddon G: Obstetric outcome of Bangladeshi women in east London. Br J Obstet Gynaecol 1995, 102:630-637.

43. Yoong W, Wagley A, Fong C, Chukwuma C, Nauta M: Obstetric performance of ethnic Kosovo Albanian asylum seekers in London: a case-control study. J Obstet Gynaecol 2004, 24:510-512.

44. Yoong W, Kolhe S, Karoshi M, Ullah M, Nauta M: The obstetric performance of United Kingdom asylum seekers from Somalia: a case-control study and literature review. Int J Fertility \& Womens Med 2005, 50:175-179.

45. Small R, Gagnon A, Gissler M, Zeitlin J, Bennis M, Glazier RH, Haelterman E, Martens G, McDermott S, Urquia M, et al: Somali women and their pregnancy outcomes post-migration: data from six receiving countries. Br J Obstet Gynaecol 2008, 115:1630-1640.

46. Malin M, Gissler M: Maternal care and birth outcomes among ethnic minority women in Finland. BMC Public Health 2009, 9:84.

47. Fedeli U, Alba N, Lisiero M, Zambon F, Avossa F, Spolaore P: Obstetric hospitalizations among Italian women, regular and irregular immigrants in North Eastern Italy. Acta Obstet Gynecol Scand 2010, 89:1432-1437.

48. Gann P, Nghiem L, Warner S: Pregnancy characteristics and outcomes of Cambodian refugees. Am J Public Health 1989, 79:1251-1257.

49. Howell R: Philippino and Vietnamese Women - A Study of Intrapartum Differences. Aust N Z J Public Health 1989, 29:399-402.

50. Mossialos E, Allin S, Karras K, Davaki K: An investigation of Caesarean sections in three Greek hospitals. Eur J Public Health 2005, 15:288-295.

51. Helsel D, Petitti DB, Kunstadter P: Pregnancy among the Hmong: birthweight, age, and parity. Am J Public Health 1992, 82:1361-1364.
52. Chan A, Roder D, Macharper T: Obstetric Profiles of Immigrant Women from Non-English Speaking Countries in South Australia, 1981-1983. Aust N Z J Obstet Gynaecol 1988, 28:90-95.

53. Rio I, Castelló A, Barona C, Jané M, Más R, Rebagliato M, Bosch S, Martínez E, Bolúmar F: Caesarean section rates in immigrant and native women in Spain: the importance of geographical origin and type of hospital for delivery. Eur J Public Health 2010, 20:524-529.

54. Triantafyllidis G, Tziouva K, Papastefanou I, Samolis S, Katsetos C, Panagopoulos P: Frequency of Cesarean Section (CS) and Infectious Diseases Among Greek and Immigrant Women in a Greek Public Nursery [abstract]. Abstracts of the XXII European Congress of Perinatal Medicine, 2010. J Matern Fetal Neonatal Med 2010, 23:289.

55. Zlot Al, Jackson DJ, Korenbrot C: Association of acculturation with cesarean section among Latinas. Matern Child Health J 2005, 9:11-20

56. Berger C, Liska G, Gallier J, Soutoul JH: Comportement obstetrical des migrantes. Revue de Medecine de Tours 1973, 7:607-611.

57. Forna F, Jamieson DJ, Sanders D, Lindsay MK: Pregnancy outcomes in foreign-born and US-born women. Int J Gynaecol Obstet 2003, 83:257-265.

58. Hazekamp JT: Care and control in immigrant Pakistani pregnancy. Experience from Ullevaal Hospital in Oslo. Tidsskrift for den Norske laegeforening: tidsskrift for praktisk medicin, ny raekke 1982, 102:237-239.

59. Richman D, Dixon S: Comparative study of Cambodian, Hmong, and Caucasian infant and maternal perinatal profiles. J Nurse Midwifery 1985, 30:313-319.

60. Henry OA, Guaran RL, Petterson CD, Walstab JE: Obstetric and birthweight differences between Vietnam-born and Australian-born women. Med J Australia 1992, 156:321-324.

61. Small R, Lumley J: Variations in Caesarean Section by Maternal Country of Birth, Victoria 1999-2003[abstract]. J Paediatr Child Health 2007, 43:A53.

62. Zuppa AA, Orchi C, Calabrese V, Verrillo G, Perrone S, Pasqualini P, Cota F, Ranno O, Valentini P, Giannantonio C: Maternal and neonatal characteristics of an immigrant population in an Italian hospital. J Matern Fetal Neonatal Med 2010, 23:627-632.

63. Aurelius G, Ryde-Blomqvist E: Pregnancy and Delivery among Immigrants. Scand J Public Health 1978, 6:43-48.

64. Gagnon AJ, Dougherty G, Platt RW, Wahoush O, George A, Stanger E, Oxman-Martinez J, Saucier JF, Merry L, Stewart DE: Refugee and refugeeclaimant women and infants post-birth: migration histories as a predictor of Canadian health system response to needs. Can J Public Health 2007, 98:287-291.

65. Teixeira C, Correia S, Barros H: Geographic origin and risk of cesarean section in Portugal [abstract]. In Abstract Book of the 3rd Conference on Migrant and Ethnic Minority Health in Europe. Hungary: Pécs; 2010:78. 27-29 May 2010.

66. Gagnon AJ, Wahoush O, Dougherty G, Saucier JF, Dennis CL, Merry L, Stanger E, Stewart DE: The Childbearing Health and Related Service Needs of Newcomers (CHARSNN) study protocol. BMC Pregnancy Childbirth 2006, 6.

67. Loew D, Schrank P: Bericht uber 7000 Geburten in einem mittleren Krankenhaus unter Berucksichtigung der Geburtsverlaufe bei Auslanderinnen. Zentralb/ Gynakol 1966, 1:23-31.

68. Schliemann F, Schliemann G: Deliveries in foreign women. Geburtshilfe Frauenheilkd 1975, 35:210-217.

69. Saurwein A: Delivery in foreign women: frequency and indication of cesarean section. Geburtshilfe Frauenheilkd 1969, 29:728-734.

70. Harlap S, Kaufman R, Prywes R, Davies AM, Sterk W, Weiskopf P: Patterns of obstetric intervention in a total population. A report from the Jerusalem perinatal study. Israel J Med Sci 1971, 7:1115-1127.

71. Maslovitz S, Kupferminc MJ, Lessing JB, Many A: Perinatal outcome among non-residents in Israel. Israel Med Assoc J: Imaj 2005, 7:315-319.

72. Barron SL, Vessey MP: Immigration a new social factor in obstetrics. $\mathrm{Br}$ Med J 1966, 1:1189-1194.

73. Kaminski M: La grossesse des femmes migrantes à Paris. Rev Fr Gynecol Obstet 1975, 70:483-491.

74. Rudman A, El-Khouri B, Waldenström U: Evaluating multi-dimensional aspects of postnatal hospital care. Midwifery 2008, 24:425-441.

75. Press F, Katz M, Leiberman JR, Shoham I, Glezerman M: Obstetric performance in Ethiopian immigrants compared with Israeli parturients. Israel J Med Sci 1993, 29:403-407.

76. Moscioni P, Romagnoli C, Pomili G, Gilardi G: Immigrant women and health services. Experience with hospital obstetric-gynecologic department (1992-94). Minerva Ginecol 1995, 47:373-379. 
77. Walsh J, Robson M, Foley M: The influence of ethnicity on caesarean section rates in the home of active management of labour[abstract]. Int $J$ Gynecology \& Obsterics 2009, 107S2:373-374.

78. van Enk A, Doornbos HP, Nordbeck HJ: Some characteristics of labor in ethnic minorities in Amsterdam. Int J Gynaecol Obstet 1990, 33:307-311.

79. van Enk WJ, Gorissen WH, van Enk A: Teenage pregnancy and ethnicity in The Netherlands: frequency and obstetric outcome. Eur J Contracept Reprod Health Care 2000, 5:77-84

80. Schultze-Naumburg R, Scholtes G: Entbindungen bei Ausländerinnen. In Medizinische Klinik 1976, 2:63-67.

81. Janevic T: Differences in cesarean delivery in New York City by race, ethnicity, and nativity, 1995-2003 [abstract]. In Final Program for the Society for Pediatric and Perinatal Epidemiologic Research (SPER) 24th annual meeting: June 20 - 21, 2011. Montreal; 2011:74

82. Sletten K: Perinatal outcomes of immigrants giving birth at a low risk central hospital in Norway: A comparison study at Bærum hospital of different ethnic groups and Norwegian women. In Thesis. Institute of Health and Society, Health Sciences; Unpublished (completed 2011). Abstract available at: https://www.duo.uio.no/ handle/123456789/28440

83. Stray-Pedersen B, Austveg B: Our new country men: challenges and opportunities. In Mid-life Norwegian Gynaecological Association 50 years festschrift. Edited by Bordahl P, et al. Tapir Trondheim; 1996

84. Parsons L, Macfarlane AJ, Golding J: Pregnancy, birth and maternity care. In Race and health in contemporary Britain. Edited by Ahmad WIU. Buckingham: Open University Press; 1993.

85. Holan S, Vangen S, Hanssen K, Stray-Pedersen B: Diabetes hos gravide født i Asia, Afrika og Norge. Tidsskr Nor Legeforen 2008, 128:1289-1292.

86. Oliva GC, Zannella MP, Filidi C, Cavaliere AF, Casarella L, Mancuso S: Characteristics of the puerperium and complications in the vaginal delivery and the caesarean section. Comparison between the Italian and foreign population in casuistry 2000-2004 of the Policlinico "A. Gemelli". Giornale Italiano di Ostetricia e Ginecologia 2007, 29:75-81.

87. Ismail Kl, Marchocki Z, Brennan DJ, O'Donoghue K: Intrapartum caesarean rates differ significantly between ethnic groups-Relationship to induction. Eur J Obstet Gynecol Reprod Biol 2011, 158:214-219.

88. Zanconato G, lacovella C, Parazzini F, Bergamini V, Franchi M: Pregnancy outcome of migrant women delivering in a public institution in northern Italy. Gynecol Obstet Invest 2011, 72:157-162.

89. Comas M, Català L, Sala M, Payà A, Sala A, Del Amo E, Castells X, Cots F: Descriptive analysis of childbirth healthcare costs in an area with high levels of immigration in Spain. BMC Health Serv Res 2011, 11:77.

90. Giani U, Bruzzese D, Pugliese A, Saporito M, Triassi M: Risk factors analysis for Elective Caesarean Section in Campania region (Italy). Epidemiol Prev 35:101-110.

91. Von Katterfeld B, Li J, McNamara B, Langridge AT: Obstetric profiles of foreign-born women in Western Australia using data linkage, 19982006. Aust N Z J Obstet Gynaecol 2011, 51:225-232.

92. Lansakara N, Brown SJ, Gartland D: Birth outcomes, postpartum health and primary care contacts of immigrant mothers in an Australian nulliparous pregnancy cohort study. Matern Child Health J 2010, 14:807-816.

93. Shah D, Tay A, Desai A, Parikh M, Nauta M, Yoong W: The obstetric performance of Chinese immigrants residing in the UK. J Obstet Gynaecol 2011, 31:480-482.

94. Saurel-Cubizolles M-J, Saucedo M, Drewniak N, Blondel B, Bouvier-Colle M-H: Santé périnatale des femmes étrangères en France. Bulletin épidémiologique hebdomadaire 2012, 2-3-4:30-34.

95. Shah RR, Ray JG, Taback N: Adverse Pregnancy Outcomes Among Foreign-Born Canadians. J Obstet Gynaecol Can 2011, 33:207-215.

96. Walsh J, Mahony R, Armstrong F, Ryan G, O'Herlihy C, Foley M: Ethnic variation between white European women in labour outcomes in a setting in which the management of labour is standardised-a healthy migrant effect? BJOG 2011, 118:713-718.

97. Kingston D, Heaman M, Chalmers B, Kaczorowski J, O'Brien B, Lee L, Dzakpasu S, O'Campo P: Comparison of Maternity Experiences of Canadian-Born and Recent and Non-Recent Immigrant Women: Findings From the Canadian Maternity Experiences Survey. J Obstet Gynaecol Can 2011, 33:1105-1115.

98. Berger C, Laugier J, Soutoul JH: Caracteristiques de l'accouchement et du nouveau-ne de migrante: fitude de 800 dossiers. Journal de Gynicologie Obstetrique et Biologie de la Reproduction 1974, 3:1227-1234
99. Shah RR: Adverse Obstetrical and Perinatal Outcomes Among ForeignBorn Women Living in Canada. In Thesis. University of Toronto, Graduate Department of Health Policy, Management and Evaluation; 2007.

100. Ryan G, Armstrong F, Walsh J, Foley M: Ethnic variation in the mode of onset nulliparous term labour and resultant caesarean section rates with consideration of birth weight [abstract]. Ir J Med Sci 2010, 179 (supplement 2):S84.

101. Walsh J, Mahony R, McAuliffe F, O'Herlihy C, Robson M, Foley M: Ethnic variation in the onset of spontaneous nulliparous term labor and cesarean delivery rates may be explained by differences in maternal ageand BMI (319) [abstract]. Am J Obstet Gynecol 2009, 201:S127.

102. Ismail K, Marchocki Z, Brennan D, O'Donoghue K: Comparing labour outcomes between different racial groups [abstract]. Arch Dis Child Fetal Neonatal Ed 2010, 95(Suppl 1):Fa77.

103. Gagnon AJ, Van Hulst A, Merry L, George A, Saucier JF, Stanger E, Wahoush $\mathrm{O}$, Stewart DE: Cesarean section rate differences by migration indicators Archives of Gynecology and Obstetrics 2012. doi:10.1007/s00404-012-2609-7. published online November 2012

104. The HAPO Study Cooperative Research Group: Hyperglycemia and adverse pregnancy outcomes. N Engl J Med 2008, 358:1991-2002.

105. Benjamin SJ, Daniel AB, Kamath A, Ramkumar V: Anthropometric measurements as predictors of cephalopelvic disproportion. Acta Obstet Gynecol Scand 2012, 91:122-127.

106. Gagnon AJ, McDermott S, Rigol-Chachamovich J, Bandyopadhyay M, StrayPedersen B, Stewart D, for the ROAM Collaboration: International migration and gestational diabetes mellitus: a systematic review of the literature and meta-analysis. Paediatr Perinat Epidemiol 2011, 25:575-592.

107. Konje JC, Ladipo OA: Nutrition and obstructed labor. Am J Clin Nutr 2000 72:291S-2975

108. Siega-Riz AM, Laraia B: The implications of maternal overweight and obesity on the course of pregnancy and birth outcomes. Matern Child Health J 2006, 10:153-156.

109. Rosenberg TJ, Garbers S, Chavkin W, Chiasson MA: Prepregnancy weight and adverse perinatal outcomes in an ethnically diverse population. Obstet Gynecol 2003, 102:1022-1027.

110. Loetscher KCQ, Selvin S, Zimmermann R, Abrams B: Ethnic-cultural background, maternal body size and pregnancy outcomes in a diverse Swiss cohort. Women Health 2007, 45:25-40.

111. Heaman M, Bayrampour H, Kingston D, Blondel B, Gissler M, Roth C, Alexander S, Gagnon A: Migrant women's utilization of prenatal care: a systematic review. Matern Child Health J 2012, doi:10.1007/s10995-0121058-z. published online June 2012.

112. Bischoff A, Bovier PA, Isah R, Francoise G, Ariel E, Louis L: Language Barriers Between Nurses and Asylum Seekers: Their Impact on Symptom Reporting and Referral. Soc Sci Med 2003, 57:503-512.

113. US Department of Health and Human Services: Healthy people, Tracking. Washington D.C.: US Government Printing Office; 2010:2000.

114. Carballo M, Grocutt M, Hadzihasanovic A: Women and migration: a public health issue. World Health Stat Q 1996, 49:158-164.

115. Kahler LR, Sobota CM, Hines CK, Griswold K: Pregnant women at risk: An evaluation of the health status of refugee women in Buffalo, New York. Health Care Women Int 1996, 17:15-23.

116. McGinn T: Reproductive health of war-affected populations: what do we know? Int Fam Plan Perspect 2000, 26:174-180.

117. Liu J, Kerr D: Family change and economic well-being in canada: the case of recent immigrant families with children. Int Migr 2003, 41:113-140.

118. Blume K, Gustafsson B, Pedersen PPJ, Verner M: At the lower end of the table: determinants of poverty among immigrants to Denmark and Sweden. J Ethnic Migr Stud 2007, 33:373-396.

119. Liamputtong P, Watson LF: The meanings and experiences of cesarean birth among Cambodian, Lao and Vietnamese immigrant women in Australia. Women Health 2006, 43:63-82.

120. Vangen S, Johansen RE, Sundby J, Traeen B, Stray-Pedersen B: Qualitative study of perinatal care experiences among Somali women and local health care professionals in Norway. Eur J Obstet Gynecol Reprod Biol 2004, 112:29-35.

121. Hodnett ED, Fredericks S, Weston J: Support during pregnancy for women at increased risk of low birthweight babies. Cochrane Database Syst Rev 2010, 6 .

122. Hodnett ED, Gates S, Hofmeyr GJ, Sakala C, Weston J: Continuous support for women during childbirth. Cochrane Database Syst Rev 2011, 2. 
123. Bollini P, Pampallona S, Wanner P, Kupelnick B: Pregnancy Outcome of Migrant Women and Integration Policy: A Systematic Review of the International Literature. Soc Sci Med 2009, 68:452-461.

124. Gagnon AJ, Zimbeck M, Zeitlin J: Migration and perinatal health surveillance: an international Delphi survey. Eur J Obstet Gynaecol Reprod Biol 2010, 149:37-43.

125. Gissler M, Alexander S, MacFarlane A, Small R, Stray-Pedersen B, Zeitlin J, Zimbeck M, Gagnon AJ: Stillbirths and infant deaths among migrants in industrialised countries. Acta Obstet Gynecol Scand 2009, 88:134-148.

126. Urquia M, Glazier R, Blondel B, Zeitlin J, Gissler M, McFarlane A, Ng E, Heaman M, Stray-Pedersen B, Gagnon AJ: International migration and adverse birth outcomes: role of ethnicity, region of origin and destination. J Epidemiol Community Health 2010, 64:243-251.

\section{doi:10.1186/1471-2393-13-27}

Cite this article as: Merry et al:: International migration and caesarean birth: a systematic review and meta-analysis. BMC Pregnancy and Childbirth 2013 13:27.

\section{Submit your next manuscript to BioMed Central and take full advantage of:}

- Convenient online submission

- Thorough peer review

- No space constraints or color figure charges

- Immediate publication on acceptance

- Inclusion in PubMed, CAS, Scopus and Google Scholar

- Research which is freely available for redistribution 